\title{
Mathematical model of a stock market
}

\author{
N.S.Gonchar \\ Bogolubov Institute for Theoretical Physics \\ of the National Academy of Sciences of Ukraine, \\ $14^{\mathrm{b}}$ Metrolohichna Str., 252143 Kyiv, Ukraine
}

Received May 30, 2000

In this paper we construct a mathematical model of securities market. The results obtained are a good basis for an analysis of any stock market.

Key words: random process, effective stock market, option pricing

PACS: $02.50 .+s, 05.40 .+j$

Dedicated to prominent scientist Igor Yukhnovsky, initiator of my perspective research on economy.

\section{Introduction}

The aim of this paper is to propose a wide class of random processes to describe the evolution of a risk active price and to construct a mathematical theory of option pricing. For this purpose, a general mathematical model of evolution of a risk active price is proposed on a probability space constructed. On the probability space, an evolution of a risk active price is described by a random process with jumps that can have both finite and infinite number of jumps. We introduce a new notion of non-singular martingale and prove an integral representation for a wide class of local martingale by a path integral. This theorem is the basic result of the paper that permits us to introduce the important notion of an effective stock market. For an effective stock market the mathematical theory of European type options is constructed. As a result, the new formulas for option pricing, the capital investor and self-financing strategy corresponding to the minimal hedge are obtained.

\section{Some auxiliary results}

Hereafter we will use two elementary lemmas the proof of which is omitted.

Lemma 1. For any on the right continuous functions $\varphi(x)$ and $\psi(x)$, that have the 
bounded variation on $[a, b)$, the following formula

$$
\varphi(d) \psi(d)-\varphi(c) \psi(c)=\int_{(c, d]} \varphi_{-}(y) \mathrm{d} \psi(y)+\int_{(c, d]} \psi(y) \mathrm{d} \varphi(y), \quad(c, d] \subset[a, b)
$$

is valid, $F_{-}(u)=\lim _{v \uparrow u} F(v)$.

By $\mathrm{d} \varphi(y)$ and $\mathrm{d} \psi(y)$ we denoted the charges, generated by functions $\varphi(y)$ and $\psi(y)$ correspondingly, $\varphi_{-}(x)=\lim _{y \uparrow x} \varphi(y)$.

Lemma 2. The Radon-Nicodym derivative of the measure $\mathrm{d} g(y)$, generated by the function $g(y)=(1-F(y))^{-1}$, with respect to the measure $\mathrm{d} F(y)$, where $F(y)$ is on the right continuous and monotonously non-decreasing on $[a, b)$ function and such that $F(a)=0, F(x)<1, x \in[a, b), \lim _{x \rightarrow b} F(x)=1$ is given by the formula

$$
\frac{\mathrm{d} g(y)}{\mathrm{d} F(y)}=\frac{1}{(1-F(y))\left(1-F_{-}(y)\right)} \text {. }
$$

Lemma 3. For on the right continuous and monotoneously non-decreasing function $\alpha(x)$ such that $\alpha(x)<\infty, x \in[a, b), \alpha(a)=0, \lim _{x \rightarrow b} \alpha(x)=\infty$, the representation

$$
\alpha(x)=\int_{[a, x]} \frac{\mathrm{d} F(y)}{1-F_{-}(y)}
$$

is valid for a certain $F(x)$, that is on the right continuous and monotonously nondecreasing function, satisfying conditions: $F(x)<1, x \in[a, b), \lim _{x \rightarrow b} F(x)=1, F(a)=$ 0 , if and only if there exists a positive, on the right continuous and monotoneously non-decreasing solution of equation

$$
\phi(x)=\int_{[a, x]} \phi(y) \mathrm{d} \alpha(y)+1
$$

such that $\phi(a)=0, \phi(x)<\infty, x \in[a, b)$. The function $F(x)$ is given by the formula

$$
F(x)=\frac{\phi(x)-1}{\phi(x)}
$$

Proof. The necessity. By definition we put $F_{-}(y)=\lim _{x \uparrow y} F(x)$. If the representation (2) holds, then the following equality

$$
\int_{[a, x]} \frac{\mathrm{d} \alpha(y)}{1-F(y)}=\int_{[a, x]} \frac{\mathrm{d} F(y)}{(1-F(y))\left(1-F_{-}(y)\right)}=\frac{1}{1-F(x)}-1
$$


is valid. Therefore, the function

$$
\phi(x)=\frac{1}{1-F(x)}
$$

is a positive, on the right continuous and monotonously non-decreasing solution of equation (3).

The sufficiency. If there exists a solution to (3), satisfying conditions of lemma 3 , then the function (4) satisfies equation

$$
\int_{[a, x]} \frac{\mathrm{d} \alpha(y)}{1-F(y)}+1=\frac{1}{1-F(x)} .
$$

But

$$
\int_{[a, x]} \frac{\mathrm{d} F(y)}{(1-F(y))\left(1-F_{-}(y)\right)}+1=\frac{1}{1-F(x)} .
$$

The latter means that

$$
\frac{\mathrm{d} \alpha(y)}{1-F(y)}=\frac{\mathrm{d} F(y)}{(1-F(y))\left(1-F_{-}(y)\right)}
$$

or

$$
\mathrm{d} \alpha(y)=\frac{\mathrm{d} F(y)}{\left(1-F_{-}(y)\right)} .
$$

From the latter equality it follows that

$$
\alpha(x)=\int_{[a, x]} \frac{\mathrm{d} F(y)}{\left(1-F_{-}(y)\right)} .
$$

Lemma 3 is proved.

Let us give the necessary and sufficient conditions for the existence of a solution to equation (3)

Lemma 4. Nonnegative solution to the equation (3) exists if and only if the series

$$
\phi(x)=1+\sum_{n=1}^{\infty} \int_{[a, x]} \mathrm{d} \alpha\left(t_{1}\right) \int_{\left[a, t_{1}\right]} \mathrm{d} \alpha\left(t_{2}\right) \ldots \int_{\left[a, t_{n-1}\right]} \mathrm{d} \alpha\left(t_{n}\right)
$$

converges for all $x \in[a, b)$.

Proof. The necessity. If there exists a non-negative solution to (3), then this solution is the solution to the equation

$$
\phi(x)=1+\sum_{n=1}^{k} \int_{[a, x]} \mathrm{d} \alpha\left(t_{1}\right) \int_{\left[a, t_{1}\right]} d \alpha\left(t_{2}\right) \ldots \int_{\left[a, t_{n-1}\right]} \mathrm{d} \alpha\left(t_{n}\right)
$$




$$
+\int_{[a, x]} \mathrm{d} \alpha\left(t_{1}\right) \int_{\left[a, t_{1}\right]} \mathrm{d} \alpha\left(t_{2}\right) \ldots \int_{\left[a, t_{k-1}\right]} \mathrm{d} \alpha\left(t_{k}\right) \int_{\left[a, t_{k}\right]} \phi\left(t_{k+1}\right) \mathrm{d} \alpha\left(t_{k+1}\right) .
$$

From the latter equality there follows the inequality

$$
1+\sum_{n=1}^{k} \int_{[a, x]} \mathrm{d} \alpha\left(t_{1}\right) \int_{\left[a, t_{1}\right]} \mathrm{d} \alpha\left(t_{2}\right) \ldots \int_{\left[a, t_{n-1}\right]} \mathrm{d} \alpha\left(t_{n}\right) \leqslant \phi(x) .
$$

Arbitrariness of $k$, positiveness of every term of the series means the convergence of (5). The proof of sufficiency follows from the fact that if the series (5) converges then this series is evidently a solution to the equation (3). The lemma 4 is proved.

Corollary 1. If $\alpha(x)$ is a continuous and monotonously non-decreasing function, $\alpha(x)<\infty, x \in[a, b), \lim _{x \rightarrow b} \alpha(x)=\infty, \alpha(a)=0$, then the equation (3) has the solution $\phi(x)=\mathrm{e}^{\alpha(x)}$.

Corollary 2. If $\gamma(x)$ is some measurable function on $[a, b)$, which satisfies the inequality

$$
\int_{[a, x]} \gamma(y) \mathrm{d} \alpha(y)+1 \leqslant \gamma(x), x \in[a, b),
$$

then there exists a solution to equation (3).

Lemma 5. The solution to the equation (3) exists if jumps of monotonously nondecreasing and on the right continuous function $\alpha(x)$ is such that $\Delta \alpha(s) \neq 1$. It has the following form

$$
\phi(x)=\mathrm{e}^{\alpha(x)} \prod_{\{s \leqslant x\}} \frac{\mathrm{e}^{-\Delta \alpha(s)}}{(1-\Delta \alpha(s))} .
$$

If $0 \leqslant \Delta \alpha(s)<1, s \in[a, b)$, then this solution is non-negative, on the right continuous and monotonously non-decreasing function, $\Delta \alpha(s)=\alpha(s)-\alpha_{-}(s)$.

Proof. First of all the product

$$
\prod_{\{s \leqslant x\}} \frac{\mathrm{e}^{-\Delta \alpha(s)}}{(1-\Delta \alpha(s))}
$$

converges, because the estimate $\sum_{\{s \leqslant x\}} \Delta \alpha(s) \leqslant \alpha(x)<\infty, x<b$ is valid. Let us verify that $\phi(x)$ is a solution to (3) in the case when all jump points of $\alpha(x)$ are isolated points. It is sufficient to prove that if $\phi(x)$ is the solution to (3) on a certain interval $\left[a, x_{0}\right]$ and we prove that $\phi(x)$ is the solution to $(3)$ on the interval $\left(x_{0}, x\right], x>x_{0}$ then it will mean that $\phi(x)$ is the solution to $(3)$ on the interval $[a, x]$. We assume that the points $x_{i}, i=1,2, \ldots$ are the jump points of the function $\alpha(x)$. To verify that $\phi(x)$ is the solution to the equation (3) let us assume that we 
have already proved that on the interval $\left[a, x_{i}\right)$, where $x_{i}$ is the jump point of the function $\alpha(x), \phi(x)$ is the solution to equation (3), that is,

$$
\int_{\left[a, x_{i}\right)} \phi(y) \mathrm{d} \alpha(y)=\phi_{-}\left(x_{i}\right)-1=\mathrm{e}^{\alpha_{-}(x)} \prod_{\left\{s<x_{i}\right\}} \frac{\mathrm{e}^{-\Delta \alpha(s)}}{(1-\Delta \alpha(s))}-1 .
$$

Let $x$ be any point that satisfies the condition $x_{i}<x<x_{i+1}$. Since

$$
\begin{gathered}
1+\int_{[a, x]} \phi(y) \mathrm{d} \alpha(y)=1+\int_{\left[a, x_{i}\right)} \phi(y) \mathrm{d} \alpha(y)+\int_{\left[x_{i}\right]} \phi(y) \mathrm{d} \alpha(y)+\int_{\left(x_{i}, x\right]} \phi(y) \mathrm{d} \alpha(y) \\
=\phi_{-}\left(x_{i}\right)-1+\mathrm{e}^{\alpha\left(x_{i}\right)} \Delta \alpha\left(x_{i}\right) \prod_{\left\{s \leqslant x_{i}\right\}} \frac{\mathrm{e}^{-\Delta \alpha(s)}}{(1-\Delta \alpha(s))} \\
+\left[\mathrm{e}^{\alpha(x)}-\mathrm{e}^{\alpha\left(x_{i}\right)}\right] \prod_{\left\{s \leqslant x_{i}\right\}} \frac{\mathrm{e}^{-\Delta \alpha(s)}}{(1-\Delta \alpha(s))}+1=\mathrm{e}^{\alpha(x)} \prod_{\{s \leqslant x\}} \frac{\mathrm{e}^{-\Delta \alpha(s)}}{(1-\Delta \alpha(s))}=\phi(x) .
\end{gathered}
$$

To complete the proof of the lemma it is necessary to note that on the interval $\left[a, x_{1}\right)$ the solution to $(3)$ is the function $\mathrm{e}^{\alpha(x)}$. Let us prove lemma 5 in a general case. If $\alpha(x)$ satisfies the conditions to lemma 5 , then

$$
\alpha(x)=\alpha_{c}(x)+\sum_{\{s \leqslant x\}} \Delta \alpha(s)
$$

where $\alpha_{c}(x)$ is a continuous function on $[a, b)$. Let us introduce the notation

$$
\phi_{m}(x)=\mathrm{e}^{\alpha_{m}(x)} \prod_{\{s \leqslant x\}} \frac{\mathrm{e}^{-\Delta \alpha_{m}(s)}}{\left(1-\Delta \alpha_{m}(s)\right)}
$$

where

$$
\alpha_{m}(x)=\alpha_{c}(x)+\sum_{\left\{s \leqslant x, \Delta \alpha(s) \geqslant m^{-1}\right\}} \Delta \alpha(s) .
$$

In the latter sum the summation comes over all jumps of $\alpha(x)$, where the jumps of $\alpha(x)$ are greater than $m^{-1}$. It is evident that on any interval $[a, x]$ the set of such points is finite. Therefore $\phi_{m}(x)$ satisfies the equation

$$
\phi_{m}(x)=\int_{[a, x]} \phi_{m}(y) \mathrm{d} \alpha_{m}(y)+1 .
$$

Let $d<b$, then

$$
\begin{gathered}
\sup _{x \in[a, d]}\left|\phi(x)-\phi_{m}(x)\right| \leqslant \sup _{x \in[a, d]} \mathrm{e}^{\alpha_{c}(x)} \\
\times \frac{1}{\prod_{\left\{s \leqslant d, \Delta \alpha(s) \geqslant m^{-1}\right\}}(1-\Delta \alpha(s))}\left\{1-\frac{\prod_{\left\{s \leqslant d, \Delta \alpha(s) \geqslant m^{-1}\right\}}(1-\Delta \alpha(s))}{\prod_{\{s \leqslant d\}}(1-\Delta \alpha(s))}\right\} \leqslant
\end{gathered}
$$




$$
\leqslant \sup _{x \in[a, d]} \mathrm{e}^{\alpha_{c}(x)} \frac{\sum_{\left\{s \leqslant d, \Delta \alpha(s)<m^{-1}\right\}} \Delta \alpha(s)}{\prod_{\{s \leqslant d\}}(1-\Delta \alpha(s))} \rightarrow 0, \quad m \rightarrow \infty .
$$

Moreover,

$$
\operatorname{var}_{x \in[a, d]}\left[\alpha(x)-\alpha_{m}(x)\right] \leqslant \sum_{\left\{s \leqslant d, \Delta \alpha(s)<m^{-1}\right\}} \Delta \alpha(s) \rightarrow 0, \quad m \rightarrow \infty,
$$

where $\operatorname{var}_{x \in[a, d]} g(x)$ means a full variation of the function $g(x)$. From these inequalities we have

$$
\begin{gathered}
\int_{[a, x]}\left[\phi_{m}(y)-\phi(y)\right] \mathrm{d} \alpha_{m}(y) \leqslant \sup _{x \in[a, d]}\left|\phi_{m}(x)-\phi(x)\right| \underset{x \in[a, d]}{\operatorname{var}} \alpha(x) \rightarrow 0, m \rightarrow \infty, \\
\int_{[a, x]} \phi(y) \mathrm{d}\left[\alpha_{m}(y)-\alpha(y)\right] \leqslant \sup _{x \in[a, d]}|\phi(x)| \mid \underset{x \in[a, d]}{\operatorname{var}\left[\alpha_{m}(y)-\alpha(y)\right] \mid \rightarrow 0, m \rightarrow \infty .}
\end{gathered}
$$

From the equality

$$
\phi_{m}(x)=\int_{[a, x]}\left[\phi_{m}(y)-\phi(y)\right] \mathrm{d} \alpha_{m}(y)+\int_{[a, x]} \phi(y) \mathrm{d}\left[\alpha_{m}(y)-\alpha(y)\right]+\int_{[a, x]} \phi(y) \mathrm{d} \alpha(y)+1
$$

and from the preceding inequalities there follows the proof of the lemma 5 .

Theorem 1. Let $\psi(y)$ be an on the right continuous function of bounded variation on any interval $[a, x], x \in[a, b), f(y)$ be a measurable mapping with respect to the Borel $\sigma$-algebra on $[a, b)$ and bounded function on $[a, x], x \in[a, b)$. If, moreover,

$$
\alpha(x)=\int_{[a, x]} \frac{d \psi(y)}{\psi(y)-f(y)}<\infty, \quad x \in[a, b)
$$

is monotonously non-decreasing and on the right continuous function on $[a, b)$ and such that

1) $0 \leqslant \Delta \alpha(x)<1, \Delta \alpha(x)=\alpha(x)-\alpha_{-}(x), x \in[a, b)$,

2) $\lim _{x \rightarrow b} \alpha(x)=\infty, \quad \alpha(a)=0$,

3) $\lim _{\substack{x \rightarrow b \\ b}} \psi(x) \mathrm{e}^{-\alpha(x)}=0$,

4) $\int_{a}^{b}|f(x)| \mathrm{e}^{-\alpha_{-}(x)} \mathrm{d} \alpha(x)<\infty$

then for the function $\psi(x)$ the following representation

$$
\psi(x)=\frac{1}{(1-F(x))} \int_{(x, b)} f(x) \mathrm{d} F(x)
$$

is valid for a certain monotonously non-decreasing and on the right continuous function $F(x)$, such that $F(a)=0, F(x)<1, x \in[a, b), \lim _{x \rightarrow b} F(x)=1$. 
Proof. Let $F(x)$ be the function, which is constructed in the lemma 3. Let us consider the product $[1-F(x)] \psi(x)$. Then for $x<d<b$

$$
-[1-F(x)] \psi(x)+[1-F(d)] \psi(d)=\int_{(x, d]}\left[1-F_{-}(y)\right] \mathrm{d} \psi(y)-\int_{(x, d]} \psi(y) \mathrm{d} F(y) .
$$

From the lemma 3

$$
d \psi(y)=[\psi(y)-f(y)] \frac{\mathrm{d} F(y)}{\left(1-F_{-}(y)\right)}
$$

Therefore,

$$
-[1-F(x)] \psi(x)+[1-F(d)] \psi(d)=-\int_{(x, d]} f(y) \mathrm{d} F(y)
$$

Since

$$
\begin{gathered}
{[1-F(d)] \psi(d) \leqslant \mathrm{e}^{-\alpha(d)} \psi(d) \rightarrow 0, d \rightarrow b,} \\
\left|\int_{(x, d]} f(y) \mathrm{d} F(y)\right| \leqslant \int_{(x, d]}|f(y)| \mathrm{d} F(y)= \\
=\int_{(x, d]}|f(y)|\left[1-F_{-}(y)\right] \mathrm{d} \alpha(y) \leqslant \int_{(x, b)}|f(y)| \mathrm{e}^{-\alpha_{-}(y)} \mathrm{d} \alpha(y)<\infty,
\end{gathered}
$$

then, taking the limit in the equality (8), we obtain

$$
[1-F(x)] \psi(x)=\int_{(x, b)} f(y) \mathrm{d} F(y)
$$

The theorem is proved.

Theorem 2. Let $g(u)$ be a measurable function with respect to $\mathcal{B}([a, b))$ and such that

$$
\int_{[a, b)}|g(y)| \mathrm{d} F(y)<\infty
$$

then the following formula

$$
\begin{aligned}
& \frac{1}{(1-F(d))} \int_{(d, b)} g(y) \mathrm{d} F(y)-\frac{1}{(1-F(c))} \int_{(c, b)} g(y) \mathrm{d} F(y)= \\
& \quad=\int_{(c, d]}\left[\frac{1}{(1-F(u))} \int_{(u, b)} g(y) \mathrm{d} F(y)-g(u)\right] \frac{\mathrm{d} F(u)}{1-F_{-}(u)}, \quad(c, d] \subset[a, b)
\end{aligned}
$$

is valid. 
Proof. If we choose

$$
\varphi(x)=(1-F(x))^{-1}, \quad \psi(x)=\int_{(x, b)} g(u) \mathrm{d} F(u)
$$

and use lemmas 1 and 2 we obtain the proof of the theorem 2.

\section{Probability space}

Hereafter we construct a probability space, in which the securities market evolution will be considered. Let $\alpha=\left\{a_{i}^{\alpha}\right\}_{i=1}^{k(\alpha)+1}$ be a sequence from $[a, b) \subseteq R_{+}^{1}, a<b$, that satisfies conditions:

$$
a_{i}^{\alpha}<a_{i+1}^{\alpha}, \quad i=\overline{1, k(\alpha)}, \quad \bigcup_{i=1}^{k(\alpha)}\left[a_{i}^{\alpha}, a_{i+1}^{\alpha}\right)=[a, b), \quad a_{1}^{\alpha}=a, \quad a_{k(\alpha)+1}^{\alpha}=b .
$$

Therefore, the set of intervals $\left\{\left[a_{i}^{\alpha}, a_{i+1}^{\alpha}\right), i=\overline{1, k(\alpha)}\right\}$ forms a decomposition of interval $[a, b) \subseteq R_{+}^{1}$. The number $k(\alpha)$ may be both finite and infinite. Further on, we consider the family of probability spaces $\Omega_{i}=[a, b), i=\overline{1, k(\alpha)}$. On every probability space $\Omega_{i}$ a $\sigma$-algebra of events $\mathcal{F}_{i}^{0}$ is given. By definition the $\sigma$-algebra $\mathcal{F}_{i}^{0}$ is the set of subsets of $\Omega_{i}=[a, b)$, that is generated by intervals $(c, d) \subset\left[a_{i}^{\alpha}, a_{i+1}^{\alpha}\right)$. Let us determine the flow of the $\sigma$-algebras $\mathcal{F}_{i}^{0, t}, t \in[a, b), \mathcal{F}_{i}^{0, t} \subseteq \mathcal{F}_{i}^{0}$, by the formula

$$
\mathcal{F}_{i}^{0, t}= \begin{cases}\{\emptyset,[a, b)\}, & a \leqslant t \leqslant a_{i}^{\alpha} \\ \mathcal{B}\left(\left[a_{i}^{\alpha}, t\right]\right), & a_{i}^{\alpha}<t<a_{i+1}^{\alpha} \\ \bigvee_{t \in\left[a_{i}^{\alpha}, a_{i+1}^{\alpha}\right)}^{\alpha} \mathcal{B}\left(\left[a_{i}^{\alpha}, t\right]\right)=\mathcal{F}_{i}^{0}, & a_{i+1}^{\alpha} \leqslant t \leqslant b\end{cases}
$$

where we denoted by $\mathcal{B}\left(\left[a_{i}^{\alpha}, t\right]\right)$ the $\sigma$-algebra of subsets of $[a, b)$ generated by the subsets of $(c, d) \subset\left[a_{i}^{\alpha}, t\right]$ and $\underset{t \in\left[a_{i}^{\alpha}, a_{i+1}^{\alpha}\right)}{\bigvee} \mathcal{B}\left(\left[a_{i}^{\alpha}, t\right]\right)$ denotes the $\sigma$-algebra, that is the union of the $\sigma$-algebras $\mathcal{B}\left(\left[a_{i}^{\alpha}, t\right]\right)$. Let $\left\{\Omega_{\alpha}, \mathcal{F}_{\alpha}^{0}\right\}$ be the direct product of measurable spaces $\left\{\Omega_{i}, \mathcal{F}_{i}^{0}\right\}, i=\overline{1, k(\alpha)}$, and $\mathcal{F}_{t}^{0, \alpha}=\prod_{i=1}^{k(\alpha)} \mathcal{F}_{i}^{0, t}$ be the flow of the $\sigma$-algebras on the measurable space $\left\{\Omega_{\alpha}, \mathcal{F}_{\alpha}^{0}\right\}$, that is the direct product of the $\sigma$-algebras $\mathcal{F}_{i}^{0, t}$, where $\Omega_{\alpha}=\prod_{i=1}^{k(\alpha)} \Omega_{i}, \mathcal{F}_{\alpha}^{0}=\prod_{i=1}^{k(\alpha)} \mathcal{F}_{i}^{0}$. Let us determine a certain measurable space $\left\{\Omega, \mathcal{F}^{0}\right\}$. Denote by $X$ a set of sequences $\alpha=\left\{a_{i}^{\alpha}\right\}_{i=1}^{k(\alpha)+1}$ from $[a, b)$ that generate decomposition of $[a, b)$. Let $\Omega=\sum_{\alpha \in X} \bar{\Omega}_{\alpha}$ be the direct sum of the probability spaces $\bar{\Omega}_{\alpha}=\left\{\alpha, \Omega_{\alpha}\right\}$. Elements of $\bar{\Omega}_{\alpha}$ are the pairs $\left\{\alpha, \omega_{\alpha}\right\}$, where $\omega_{\alpha} \in \Omega_{\alpha}$ Let us denote by $\overline{\mathcal{F}}_{\alpha}^{0}$ the $\sigma$-algebra of events of the kind $\bar{A}_{\alpha}=\left\{\alpha, A_{\alpha}\right\}$, where $A_{\alpha} \in \mathcal{F}_{\alpha}^{0},\left\{\alpha, A_{\alpha}\right\}=$ $=\left\{\left\{\alpha, \omega_{\alpha}\right\}, \omega_{\alpha} \in A_{\alpha}\right\}$. Analogously, $\overline{\mathcal{F}}_{t}^{0, \alpha}$ is the flow of the $\sigma$-algebras from $\bar{\Omega}_{\alpha}$ of the sets of the kind $\left\{\alpha, A_{\alpha}\right\}$, where $A_{\alpha} \in \mathcal{F}_{t}^{0, \alpha}$. It is evident that $\bar{\Omega}_{\alpha} \cap \bar{\Omega}_{\beta}=\emptyset, \alpha \neq \beta$. 
Let $\Sigma$ be the $\sigma$-algebra of all subsets of $X$. Introduce a $\sigma$-algebra $\mathcal{F}^{0}$ and the flow of the $\sigma$-algebras $\mathcal{F}_{t}^{0}$ in $\Omega$. We assume that the $\sigma$-algebra $\mathcal{F}^{0}$ in $\Omega$ is the set of the subsets of the kind

$$
C_{Y}=\bigcup_{\alpha \in Y} B_{\alpha}, Y \in \Sigma, B_{\alpha} \in \overline{\mathcal{F}}_{\alpha}^{0}
$$

This follows from the following inclusions

$$
\bigcup_{i=1}^{\infty} C_{Y_{i}}=\bigcup_{\alpha \in \bigcup_{i=1}^{\infty} Y_{i}} B_{\alpha} \in \mathcal{F}^{0}, \quad \bigcap_{i=1}^{\infty} C_{Y_{i}}=\bigcup_{\alpha \in \bigcap_{i=1}^{\infty} Y_{i}} B_{\alpha} \in \mathcal{F}^{0}, \quad C_{Y_{1}} \backslash C_{Y_{2}}=\bigcup_{\alpha \in Y_{1} \backslash Y_{2}} B_{\alpha} \in \mathcal{F}^{0} .
$$

By analogy with the construction of the $\sigma$-algebra $\mathcal{F}^{0}$, the flow of the $\sigma$-algebra $\mathcal{F}_{t}^{0} \subseteq \mathcal{F}^{0}$ is the set of the subsets of the type

$$
C_{Y}=\bigcup_{\alpha \in Y} B_{\alpha}, Y \in \Sigma, B_{\alpha} \in \overline{\mathcal{F}}_{t}^{0, \alpha}
$$

Further on we deal with the measurable space $\left\{\Omega, \mathcal{F}^{0}\right\}$ and the flow of the $\sigma$ algebras $\mathcal{F}_{t}^{0} \subseteq \mathcal{F}^{0}$ on it. Hereafter we construct the probability space $\left\{\Omega, \mathcal{F}^{0}, P\right\}$.

Define a probability measure $P_{\alpha}$ on the measurable space $\left\{\Omega_{\alpha}, \mathcal{F}_{\alpha}^{0}\right\}$. For this purpose on every measurable space $\left\{\Omega_{i}, \mathcal{F}_{i}^{0}\right\}$ we determine the family of distribution functions $F_{i}^{\alpha}\left(\omega_{i}^{\alpha} \mid\left\{\omega_{\alpha}\right\}_{i-1}\right)$, that at every fixed $\left\{\omega_{\alpha}\right\}_{i-1} \in \Omega^{i-1}=\prod_{s=1}^{i-1} \Omega_{s}$ is on the right continuous and non-decreasing function of the variable $\omega_{i}^{\alpha} \in[a, b)$,

$$
F_{i}^{\alpha}\left(\omega_{i}^{\alpha} \mid\left\{\omega_{\alpha}\right\}_{i-1}\right)=\left\{\begin{array}{lll}
0, & a \leqslant \omega_{i}^{\alpha} \leqslant a_{i}^{\alpha}, & \left\{\omega_{\alpha}\right\}_{i-1} \in \Omega^{i-1} \\
\phi_{i}^{\alpha}\left(\omega_{i}^{\alpha} \mid\left\{\omega_{\alpha}\right\}_{i-1}\right), & a_{i}^{\alpha}<\omega_{i}^{\alpha}<a_{i+1}^{\alpha}, & \left\{\omega_{\alpha}\right\}_{i-1} \in \Omega^{i-1} \\
1, & a_{i+1}^{\alpha} \leqslant \omega_{i}^{\alpha}<b, & \left\{\omega_{\alpha}\right\}_{i-1} \in \Omega^{i-1}
\end{array}\right.
$$

where $\left\{\omega_{\alpha}\right\}_{i-1}=\left\{\omega_{1}^{\alpha}, \ldots, \omega_{i-1}^{\alpha}\right\}, \omega_{\alpha}=\left\{\omega_{1}^{\alpha}, \ldots, \omega_{k(\alpha)}^{\alpha}\right\}$.

The function $\phi_{i}^{\alpha}\left(\omega_{i}^{\alpha} \mid\left\{\omega_{\alpha}\right\}_{i-1}\right)$ satisfies the conditions: $0 \leqslant \phi_{i}^{\alpha}\left(\omega_{i}^{\alpha} \mid\left\{\omega_{\alpha}\right\}_{i-1}\right)<1$, it is on the right continuous and non-decreasing function of the variable $\omega_{i}^{\alpha}$ on $\left[a_{i}^{\alpha}, a_{i+1}^{\alpha}\right)$ at every fixed $\left\{\omega_{\alpha}\right\}_{i-1} \in \Omega^{i-1}$, moreover, it is a measurable function from the measurable space $\left\{\Omega^{i-1}, \overline{\mathcal{F}}_{i-1}^{0}\right\}$ to the measurable space $\{[0,1], \mathcal{B}([0,1])\}$ at every fixed $\omega_{i}^{\alpha}$, where $\mathcal{B}([0,1])$ is the Borel $\sigma$-algebra on $[0,1], \overline{\mathcal{F}}_{i-1}^{0}=\prod_{s=1}^{i-1} \mathcal{F}_{s}^{0}$. Denote by $F_{i}^{\alpha}\left(\mathrm{d} \omega_{i}^{\alpha} \mid\left\{\omega_{\alpha}\right\}_{i-1}\right)$ the measure constructed by the distribution function $F_{i}^{\alpha}\left(\omega_{i}^{\alpha} \mid\left\{\omega_{\alpha}\right\}_{i-1}\right)$ on the $\sigma$-algebra $\mathcal{F}_{i}^{0}$ at every fixed $\left\{\omega_{\alpha}\right\}_{i-1} \in \Omega^{i-1}$. It is evident that $F_{i}^{\alpha}\left(\mathrm{d} \omega_{i}^{\alpha} \mid\left\{\omega_{\alpha}\right\}_{i-1}\right)$ is concentrated on the subset $\left[a_{i}^{\alpha}, a_{i+1}^{\alpha}\right) \subset \Omega_{i}$. Let us determine a measure on the probability space $\left\{\Omega_{\alpha}, \mathcal{F}_{\alpha}^{0}\right\}$, having determined it on the set of the type $A_{1} \times \ldots \times A_{k(\alpha)}, A_{i} \in \mathcal{F}_{i}^{0}$ by the formula

$$
\begin{gathered}
P_{\alpha}\left(A_{1} \times \ldots \times A_{k(\alpha)}\right)= \\
=\int_{A_{1}} \ldots \int_{A_{k(\alpha)}} F_{1}^{\alpha}\left(\mathrm{d} \omega_{1}^{\alpha}\right) F_{2}^{\alpha}\left(\mathrm{d} \omega_{2}^{\alpha} \mid\left\{\omega_{\alpha}\right\}_{1}\right) \times \ldots \times F_{k(\alpha)}^{\alpha}\left(\mathrm{d} \omega_{k(\alpha)}^{\alpha} \mid\left\{\omega_{\alpha}\right\}_{k(\alpha)-1}\right) .
\end{gathered}
$$


The function of the sets so defined can be extended to a certain measure $P_{\alpha}$ on $\mathcal{F}_{\alpha}^{0}$ due to Ionescu and Tulcha theorem [1]. We put by definition that on the $\sigma$-algebra $\overline{\mathcal{F}}_{\alpha}^{0}$ the probability measure $\bar{P}_{\alpha}$ is given by the formula $\bar{P}_{\alpha}\left(\bar{A}_{\alpha}\right)=P_{\alpha}\left(A_{\alpha}\right)$. Further on we consider both the probability spaces $\left\{\Omega_{\alpha}, \mathcal{F}_{\alpha}^{0}, P_{\alpha}\right\}$ and the probability spaces $\left\{\bar{\Omega}_{\alpha}, \overline{\mathcal{F}}_{\alpha}^{0}, \bar{P}_{\alpha}\right\}$, that are isomorphic, and the flows of the $\sigma$-algebras $\mathcal{F}_{t}^{0, \alpha} \subseteq \mathcal{F}_{\alpha}^{0}$ and $\overline{\mathcal{F}}_{t}^{0, \alpha} \subseteq \overline{\mathcal{F}}_{\alpha}^{0}$ on the spaces $\Omega_{\alpha}$ and $\bar{\Omega}_{\alpha}$ correspondingly. If $\mu(Y)$ is a probability measure on $\Sigma$, we put that on the $\sigma$-algebra $\mathcal{F}^{0}$ the probability measure $P$ is given by the formula

$$
P\left(C_{Y}\right)=\int_{Y} \bar{P}_{\alpha}\left(B_{\alpha}\right) \mathrm{d} \mu(\alpha), \quad C_{Y}=\bigcup_{\alpha \in Y} B_{\alpha}, \quad Y \in \Sigma, \quad B_{\alpha} \in \overline{\mathcal{F}}_{\alpha}^{0} .
$$

The latter integral exists, because $\bar{P}_{\alpha}\left(B_{\alpha}\right)$ is a measurable mapping from the measurable space $\{X, \Sigma\}$ to the measurable space $\left\{R^{1}, \mathcal{B}\left(R^{1}\right)\right\}$, where $\mathcal{B}\left(R^{1}\right)$ is the Borel $\sigma$-algebra on $R^{1}$.

Further on we consider the probability space $\left\{\Omega, \mathcal{F}^{0}, P\right\}$ and the flow of the $\sigma$-algebras $\mathcal{F}_{t}^{0} \subseteq \mathcal{F}^{0}$ on it, the probability space $\{\Omega, \mathcal{F}, P\}$ and the flow of the $\sigma$ algebras $\mathcal{F}_{t} \subseteq \mathcal{F}$, where $\mathcal{F}$ and $\mathcal{F}_{t}$ are the completion of $\mathcal{F}^{0}$ and $\mathcal{F}_{t}^{0}$ correspondingly with respect to the measure $P$. Then we use the same notation $P$ for the extension of a measure $P$ from the $\sigma$-algebra $\mathcal{F}^{0}$ onto the $\sigma$-algebra $\mathcal{F}$, where the $\sigma$-algebra $\mathcal{F}$ is the completion of the $\sigma$-algebra $\mathcal{F}^{0}$ by the sets of zero measure with respect to the measure $P$ given on the $\sigma$-algebra $\mathcal{F}^{0}$.

\section{Random processes on the probability space}

Definition 1. A consistent with the flow of the $\sigma$-algebras $\mathcal{F}_{t}^{0}$ measurable mapping $\zeta_{t}\left(\left\{\alpha, \omega_{\alpha}\right\}\right)$ from the measurable space $\left\{\Omega, \mathcal{F}^{0}\right\}$ to the measurable space $\left\{R^{1}, \mathcal{B}\left(R^{1}\right)\right\}$ belongs to a certain class $K$ if for $\zeta_{t}\left(\left\{\alpha, \omega_{\alpha}\right\}\right)$ the representation

$$
\begin{aligned}
\zeta_{t}\left(\left\{\alpha, \omega_{\alpha}\right\}\right)= & \sum_{i=1}^{k(\alpha)} \chi_{\left[a_{i}^{\alpha}, a_{i+1}^{\alpha}\right)}(t) \zeta_{t}^{i, \alpha}\left(\left\{\omega_{\alpha}\right\}_{i}\right), \\
\zeta_{t}^{i, \alpha}\left(\left\{\omega_{\alpha}\right\}_{i}\right)= & f_{i}^{\alpha}\left(\left\{\omega_{\alpha}\right\}_{i}\right) \chi_{\left[a_{i}^{\alpha}, t\right]}\left(\omega_{i}^{\alpha}\right)+\psi_{i}^{\alpha}\left(\left\{\omega_{\alpha}\right\}_{i-1}, t\right) \chi_{\left(t, a_{i+1}^{\alpha}\right)}\left(\omega_{i}^{\alpha}\right), \\
& t \in\left[a_{i}^{\alpha}, a_{i+1}^{\alpha}\right)
\end{aligned}
$$

is valid, where $f_{i}^{\alpha}\left(\left\{\omega_{\alpha}\right\}_{i}\right)$ is a measurable mapping from the measurable space $\left\{\Omega^{i}, \overline{\mathcal{F}}_{i}^{0}\right\}$ to the measurable space $\left\{R^{1}, \mathcal{B}\left(R^{1}\right)\right\}$ at every fixed $\alpha \in X^{0}, i=\overline{1, k(\alpha)}$, $\psi_{i}^{\alpha}\left(\left\{\omega_{\alpha}\right\}_{i-1}, t\right)$ is a measurable mapping from the measurable space $\left\{\Omega^{i-1}, \overline{\mathcal{F}}_{i-1}^{0}\right\}$ to the measurable space $\left\{R^{1}, \mathcal{B}\left(R^{1}\right)\right\}$ at every fixed $t \in\left[a_{i}^{\alpha}, a_{i+1}^{\alpha}\right), \alpha \in X^{0}, i=\overline{2, k(\alpha)}$.

Further we deal with the space $X^{0}$ that consists of sequences $\alpha=\left\{a_{i}^{\alpha}\right\}_{i=1}^{k(\alpha)+1}$ not having limiting points on the interval $[a, x], \forall x<b, \Sigma$ is the $\sigma$-algebra of all subsets of $X^{0}$. Hereinafter $\chi_{D}(t)$ denotes the indicator function of the set $D$ from $[a, b)$. 
Definition 2. By $K^{0}$ we denote the subclass of the class $K$ of measurable mappings, satisfying conditions:

1) $\psi_{i}^{\alpha}\left(\left\{\omega_{\alpha}\right\}_{i-1}, t\right)$ is an on the right continuous function of bounded variation of the variable $t$ on any interval $\left[a_{i}^{\alpha}, \tau\right], \tau \in\left[a_{i}^{\alpha}, a_{i+1}^{\alpha}\right)$ at every fixed $\left\{\omega_{\alpha}\right\}_{i-1} \in \Omega^{i-1}, i=$ $=\overline{1, k(\alpha)}$.

2) $f_{i}^{\alpha}\left(\left\{\omega_{\alpha}\right\}_{i}\right)$ is a measurable and bounded mapping from the measurable space $\left\{\Omega^{i}, \overline{\mathcal{F}}_{i}^{0}\right\}$ to the measurable space $\left\{R^{1}, \mathcal{B}\left(R^{1}\right)\right\}$.

3) The function

$$
\gamma^{i, \alpha}\left(\left\{\omega_{\alpha}\right\}_{i-1}, t\right)=\int_{\left[a_{i}^{\alpha}, t\right]} \frac{\psi_{i}^{\alpha}\left(\left\{\omega_{\alpha}\right\}_{i-1}, \mathrm{~d} \tau\right)}{\left[\psi_{i}^{\alpha}\left(\left\{\omega_{\alpha}\right\}_{i-1}, \tau\right)-f_{i}^{\alpha}\left(\left\{\omega_{\alpha}\right\}_{i-1}, \tau\right)\right]}, \quad i=\overline{1, k(\alpha)},
$$

where $f_{i}^{\alpha}\left(\left\{\omega_{\alpha}\right\}_{i-1}, \tau\right)=f_{i}^{\alpha}\left(\left\{\omega_{\alpha}\right\}_{i}\right) \mid \omega_{i}^{\alpha}=\tau$, is monotonously non-decreasing and on the right continuous function of the variable $t$ on the interval $\left[a_{i}^{\alpha}, a_{i+1}^{\alpha}\right)$ at every fixed $\left\{\omega_{\alpha}\right\}_{i-1} \in \Omega^{i-1}, \alpha \in X^{0}$, satisfying conditions:

a) $\Delta \gamma^{i, \alpha}\left(\left\{\omega_{\alpha}\right\}_{i-1}, t\right)<1, \quad\left\{\omega_{\alpha}\right\}_{i-1} \in \Omega^{i-1}, \quad t \in\left[a_{i}^{\alpha}, a_{i+1}^{\alpha}\right)$,

$\Delta \gamma^{i, \alpha}\left(\left\{\omega_{\alpha}\right\}_{i-1}, t\right)=\gamma^{i, \alpha}\left(\left\{\omega_{\alpha}\right\}_{i-1}, t\right)-\gamma^{i, \alpha}\left(\left\{\omega_{\alpha}\right\}_{i-1}, t_{-}\right)$,

$\gamma^{i, \alpha}\left(\left\{\omega_{\alpha}\right\}_{i-1}, t_{-}\right)=\lim _{s \uparrow t} \gamma^{i, \alpha}\left(\left\{\omega_{\alpha}\right\}_{i-1}, s\right)$.

b) $\lim _{t \rightarrow a_{i+1}^{\alpha}} \gamma^{i, \alpha}\left(\left\{\omega_{\alpha}\right\}_{i-1}, t\right)=\infty, \quad \gamma^{i, \alpha}\left(\left\{\omega_{\alpha}\right\}_{i-1}, a_{i}^{\alpha}\right)=0, \quad\left\{\omega_{\alpha}\right\}_{i-1} \in \Omega^{i-1}, \alpha \in X^{0}$.

c) $\lim _{t \rightarrow a_{i+1}^{\alpha}} \psi_{i}^{\alpha}\left(\left\{\omega_{\alpha}\right\}_{i-1}, t\right) \exp \left\{-\gamma^{i, \alpha}\left(\left\{\omega_{\alpha}\right\}_{i-1}, t\right)\right\}=0$,

d) $\int_{\left[a_{i}^{\alpha}, a_{i+1}^{\alpha}\right)}\left|f_{i}^{\alpha}\left(\left\{\omega_{\alpha}\right\}_{i-1}, t\right)\right| \exp \left\{-\gamma^{i, \alpha}\left(\left\{\omega_{\alpha}\right\}_{i-1}, t_{-}\right)\right\} \gamma^{i, \alpha}\left(\left\{\omega_{\alpha}\right\}_{i-1}, \mathrm{~d} t\right)<\infty$, $\left\{\omega_{\alpha}\right\}_{i-1} \in \Omega^{i-1}, \alpha \in X^{0}$.

We denoted by $\gamma^{i, \alpha}\left(\left\{\omega_{\alpha}\right\}_{i-1}, d t\right)$ the measure on $\mathcal{B}\left(\left[a_{i}^{\alpha}, a_{i+1}^{\alpha}\right)\right)$, generated by the monotonously non-decreasing and on the right continuous function $\gamma^{i, \alpha}\left(\left\{\omega_{\alpha}\right\}_{i-1}, t\right)$ of the variable $t$ at every fixed $\left\{\omega_{\alpha}\right\}_{i-1} \in \Omega^{i-1}, \mathcal{B}\left(\left[a_{i}^{\alpha}, a_{i+1}^{\alpha}\right)\right)$ is the Borel $\sigma$-algebra on the interval $\left[a_{i}^{\alpha}, a_{i+1}^{\alpha}\right)$.

Lemma 6. Any on the right continuous and uniformly integrable martingale on the probability space $\{\Omega, \mathcal{F}, P\}$ with respect to the flow $\mathcal{F}_{t}$ is given by the formula

$$
M_{t}\left(\left\{\alpha, \omega_{\alpha}\right\}\right)=\sum_{i=1}^{k(\alpha)} \chi_{\left[a_{i}^{\alpha}, a_{i+1}^{\alpha}\right)}(t) m_{t}^{i, \alpha}\left(\left\{\omega_{\alpha}\right\}_{i}\right),
$$

where

$$
\begin{aligned}
m_{t}^{i, \alpha}\left(\left\{\omega_{\alpha}\right\}_{i}\right)= & f_{i}^{\alpha}\left(\left\{\omega_{\alpha}\right\}_{i}\right) \chi_{\left[a_{i}^{\alpha}, t\right]}\left(\omega_{i}^{\alpha}\right)+\psi_{i}^{\alpha}\left(\left\{\omega_{\alpha}\right\}_{i-1}, t\right) \chi_{\left(t, a_{i+1}^{\alpha}\right)}\left(\omega_{i}^{\alpha}\right), \\
f_{i}^{\alpha}\left(\left\{\omega_{\alpha}\right\}_{i}\right)= & \int_{\Omega_{i+1}} \ldots \int_{\Omega_{k(\alpha)}} g^{\alpha}\left(\left\{\omega_{\alpha}\right\}_{i},\left\{\omega_{\alpha}\right\}_{[i+1, k(\alpha)]}\right) \\
& \times F_{i+1}^{\alpha}\left(\mathrm{d} \omega_{i+1}^{\alpha} \mid\left\{\omega_{\alpha}\right\}_{i}\right) \times \ldots \times F_{k(\alpha)}^{\alpha}\left(\mathrm{d} \omega_{k(\alpha)}^{\alpha} \mid\left\{\omega_{\alpha}\right\}_{k(\alpha)-1}\right), \\
\psi_{i}^{\alpha}\left(\left\{\omega_{\alpha}\right\}_{i-1}, t\right)= & \frac{1}{1-F_{i}^{\alpha}\left(t \mid\left\{\omega_{\alpha}\right\}_{i-1}\right)} \int_{\left(t, a_{i+1}^{\alpha}\right)} f_{i}^{\alpha}\left(\left\{\omega_{\alpha}\right\}_{i}\right) F_{i}^{\alpha}\left(\mathrm{d} \omega_{i}^{\alpha} \mid\left\{\omega_{\alpha}\right\}_{i-1}\right), \\
& i=\frac{1, k(\alpha)}{1, \quad \alpha \in X^{0},}
\end{aligned}
$$


$g^{\alpha}\left(\left\{\omega_{\alpha}\right\}_{i},\left\{\omega_{\alpha}\right\}_{[i+1, k(\alpha)]}\right)$ is a measurable and integrable function on the probability space $\{\Omega, \mathcal{F}, P\}$, that is, $\int_{X^{0}} M_{\alpha}\left|g\left(\left\{\alpha, \omega_{\alpha}\right\}\right)\right| \mathrm{d} \mu(\alpha)<\infty$.

Proof. Further on, for the mapping $g^{\alpha}\left(\left\{\omega_{\alpha}\right\}_{i},\left\{\omega_{\alpha}\right\}_{[i+1, k(\alpha)]}\right)$ we use one more notation $g\left(\left\{\alpha, \omega_{\alpha}\right\}\right)=g^{\alpha}\left(\left\{\omega_{\alpha}\right\}_{i},\left\{\omega_{\alpha}\right\}_{[i+1, k(\alpha)]}\right)$, where $\left\{\omega_{\alpha}\right\}_{i}=\left\{\omega_{1}^{\alpha}, \ldots, \omega_{i}^{\alpha}\right\},\left\{\omega_{\alpha}\right\}_{[i, k(\alpha)]}=$ $\left\{\omega_{i+1}^{\alpha}, \ldots, \omega_{k(\alpha)}^{\alpha}\right\}, \omega_{\alpha}=\left\{\omega_{1}^{\alpha}, \ldots, \omega_{k(\alpha)}^{\alpha}\right\}=\left\{\left\{\omega_{\alpha}\right\}_{i},\left\{\omega_{\alpha}\right\}_{[i, k(\alpha)]}\right\}$. Taking into account the $\sigma$-algebra $\Sigma$ from $X^{0}$ consists of all subsets of $X^{0}$ to prove the lemma 6 , it is sufficient to calculate the conditional expectation

$$
\left.M\left\{g\left(\left\{\beta, \omega_{\beta}\right\}\right) \mid \mathcal{F}_{t}\right\}\right|_{\beta=\alpha}=M_{\alpha}\left\{g\left(\left\{\alpha, \omega_{\alpha}\right\}\right) \mid \overline{\mathcal{F}}_{t}^{0, \alpha}\right\}
$$

where $g\left(\left\{\alpha, \omega_{\alpha}\right\}\right)$ is a measurable and integrable function on the probability space $\{\Omega, \mathcal{F}, P\}, M_{\alpha}\left\{g\left(\left\{\alpha, \omega_{\alpha}\right\}\right) \mid \overline{\mathcal{F}}_{t}^{0, \alpha}\right\}$ is the conditional expectation with respect to the flow of the $\sigma$-algebras $\overline{\mathcal{F}}_{t}^{0, \alpha} \subseteq \overline{\mathcal{F}}_{\alpha}^{0}$ on the probability space $\left\{\bar{\Omega}_{\alpha}, \overline{\mathcal{F}}_{\alpha}^{0}, \bar{P}_{\alpha}\right\}$. Suppose that $t \in\left[a_{i}^{\alpha}, a_{i+1}^{\alpha}\right)$. From this it follows that

$$
\varphi_{i}^{t}\left(\left\{\omega_{\alpha}\right\}\right)=M_{\alpha}\left\{g\left(\left\{\alpha, \omega_{\alpha}\right\}\right) \mid \overline{\mathcal{F}}_{t}^{0, \alpha}\right\}
$$

is the measurable mapping from $\left\{\bar{\Omega}_{\alpha}, \overline{\mathcal{F}}_{t}^{0, \alpha}, \bar{P}_{\alpha}\right\}$ to $\left\{R^{1}, \mathcal{B}\left(R^{1}\right)\right\}$, where $\overline{\mathcal{F}}_{t}^{0, \alpha}=$ $\prod_{s=1}^{i-1} \mathcal{F}_{s}^{0} \times \mathcal{F}_{i}^{0, t} \times \prod_{s=i+1}^{k(\alpha)} O_{s}, O_{s}=\{\emptyset,[a, b)\}, s=\overline{i+1, k(\alpha)}$. Due to the structure of the $\sigma$-algebra $\overline{\mathcal{F}}_{t}^{0, \alpha}$ it follows that $\varphi_{i}^{t}\left(\left\{\omega_{\alpha}\right\}\right)$ depends only on variables $\left\{\omega_{\alpha}\right\}_{i}$ and $\varphi_{i}^{t}\left(\left\{\omega_{\alpha}\right\}\right)$ is a measurable mapping from $\left\{\Omega^{i}, \prod_{s=1}^{i-1} \mathcal{F}_{s}^{0} \times \mathcal{F}_{i}^{0, t}\right\}$ to $\left\{R^{1}, \mathcal{B}\left(R^{1}\right)\right\}$. Granting this notation we have

$$
\begin{gathered}
\varphi_{i}^{t}\left(\left\{\omega_{\alpha}\right\}\right)=Q_{i}^{t}\left(\left\{\omega_{\alpha}\right\}_{i}\right) \\
=\varphi_{i}^{\alpha}\left(\left\{\omega_{\alpha}\right\}_{i}, t\right) \chi_{\left[a_{i}^{\alpha}, t\right]}\left(\omega_{i}^{\alpha}\right)+\psi_{i}^{\alpha}\left(\left\{\omega_{\alpha}\right\}_{i-1}, t\right) \chi_{\left(t, a_{i+1}^{\alpha}\right)}\left(\omega_{i}^{\alpha}\right) .
\end{gathered}
$$

Really,

$$
Q_{i}^{t}\left(\left\{\omega_{\alpha}\right\}_{i}\right)=Q_{i}^{t}\left(\left\{\omega_{\alpha}\right\}_{i}\right) \chi_{\left[a_{i}^{\alpha}, t\right]}\left(\omega_{i}^{\alpha}\right)+Q_{i}^{t}\left(\left\{\omega_{\alpha}\right\}_{i}\right) \chi_{\left(t, a_{i+1}^{\alpha}\right)}\left(\omega_{i}^{\alpha}\right) .
$$

Because of the fact that $Q_{i}^{t}\left(\left\{\omega_{\alpha}\right\}_{i}\right) \chi_{\left[a_{i}^{\alpha}, t\right]}\left(\omega_{i}^{\alpha}\right)$ is a measurable mapping from $\left\{\Omega^{i}, \prod_{s=1}^{i-1} \mathcal{F}_{s}^{0} \times \mathcal{F}_{i}^{0, t}\right\}$ to $\left\{R^{1}, \mathcal{B}\left(R^{1}\right)\right\}$ it follows that $Q_{i}^{t}\left(\left\{\omega_{\alpha}\right\}_{i}\right) \chi_{\left(t, a_{i+1}^{\alpha}\right)}\left(\omega_{i}^{\alpha}\right)$ is also the measurable mapping. But this is possible, when $Q_{i}^{t}\left(\left\{\omega_{\alpha}\right\}_{i}\right)$ does not depend on the variable $\omega_{i}^{\alpha} \in(t, b)$, because the only measurable sets $\prod_{s=1}^{i-1} B_{s} \times A_{i}$ belong to the $\sigma$-algebra $\prod_{s=1}^{i-1} \mathcal{F}_{s}^{0} \times \mathcal{F}_{i}^{0, t}$, when $\omega_{i}^{\alpha} \in(t, b)$, where $B_{s} \in \mathcal{F}_{s}^{0}, A_{i}=\left[a, a_{i}^{\alpha}\right) \cup(t, b)$.

Putting

$$
\begin{gathered}
Q_{i}^{t}\left(\left\{\omega_{\alpha}\right\}_{i}\right)=\varphi_{i}^{\alpha}\left(\left\{\omega_{\alpha}\right\}_{i}, t\right), \quad\left\{\omega_{\alpha}\right\}_{i} \in \Omega^{i-1} \times\left[a_{i}^{\alpha}, t\right], \\
Q_{i}^{t}\left(\left\{\omega_{\alpha}\right\}_{i}\right)=\psi_{i}^{\alpha}\left(\left\{\omega_{\alpha}\right\}_{i-1}, t\right), \quad\left\{\omega_{\alpha}\right\}_{i} \in \Omega^{i-1} \times\left\{\left[a, a_{i}^{\alpha}\right) \cup(t, b)\right\},
\end{gathered}
$$


we prove the representation (14). Taking into account the definition of the conditional expectation we have

$$
\begin{aligned}
& \int_{B_{1}} \ldots \int_{B_{i-1}} \int_{A} Q_{i}^{t}\left(\left\{\omega_{\alpha}\right\}_{i}\right) F_{1}^{\alpha}\left(\mathrm{d} \omega_{1}^{\alpha}\right) \times \ldots \times F_{i}^{\alpha}\left(\mathrm{d} \omega_{i}^{\alpha} \mid\left\{\omega_{\alpha}\right\}_{i-1}\right)= \\
& \quad=\int_{B_{1}} \ldots \int_{B_{i-1}} \int_{A} \int_{\Omega_{i+1}} \ldots \int_{\Omega_{k(\alpha)}} g\left(\left\{\alpha, \omega_{\alpha}\right\}\right) F_{1}^{\alpha}\left(\mathrm{d} \omega_{1}^{\alpha}\right) \times \ldots \times F_{k(\alpha)}^{\alpha}\left(\mathrm{d} \omega_{k(\alpha)}^{\alpha} \mid\left\{\omega_{\alpha}\right\}_{k(\alpha)-1}\right) \\
& B_{s} \in \Omega_{s}, \quad s=\overline{1, i-1}, \quad A \in \mathcal{F}_{i}^{0, t} .
\end{aligned}
$$

Let us introduce the measurable mapping

$$
\begin{gathered}
f_{i}^{\alpha}\left(\left\{\omega_{\alpha}\right\}_{i}\right)=\int_{\Omega_{i+1}} \ldots \int_{\Omega_{k(\alpha)}} g^{\alpha}\left(\left\{\omega_{\alpha}\right\}_{i},\left\{\omega_{\alpha}\right\}_{[i+1, k(\alpha)]}\right) \\
\times F_{i+1}^{\alpha}\left(\mathrm{d} \omega_{i+1}^{\alpha} \mid\left\{\omega_{\alpha}\right\}_{i}\right) \times \ldots \times F_{k(\alpha)}^{\alpha}\left(\mathrm{d} \omega_{k(\alpha)}^{\alpha} \mid\left\{\omega_{\alpha}\right\}_{k(\alpha)-1}\right)
\end{gathered}
$$

from the measurable space $\left\{\Omega^{i}, \overline{\mathcal{F}}_{i}^{0}\right\}$ to the measurable space $\left\{R^{1}, \mathcal{B}\left(R^{1}\right)\right\}$. It is evident that

$$
\int_{\Omega_{1}} \ldots \int_{\Omega_{i}}\left|f_{i}^{\alpha}\left(\left\{\omega_{\alpha}\right\}_{i}\right)\right| F_{1}^{\alpha}\left(\mathrm{d} \omega_{1}^{\alpha}\right) \times \ldots \times F_{i}^{\alpha}\left(\mathrm{d} \omega_{i}^{\alpha} \mid\left\{\omega_{\alpha}\right\}_{i-1}\right) \leqslant M_{\alpha}\left|g\left(\left\{\alpha, \omega_{\alpha}\right\}\right)\right|<\infty .
$$

From this and (15) it follows that

$$
\begin{gathered}
\varphi_{i}^{\alpha}\left(\left\{\omega_{\alpha}\right\}_{i}, t\right)=f_{i}^{\alpha}\left(\left\{\omega_{\alpha}\right\}_{i}\right), \\
\psi_{i}^{\alpha}\left(\left\{\omega_{\alpha}\right\}_{i-1}, t\right)=\frac{1}{1-F_{i}^{\alpha}\left(t \mid\left\{\omega_{\alpha}\right\}_{i-1}\right)} \int_{\left(t, a_{i+1}^{\alpha}\right)} f_{i}^{\alpha}\left(\left\{\omega_{\alpha}\right\}_{i}\right) F_{i}^{\alpha}\left(\mathrm{d} \omega_{i}^{\alpha} \mid\left\{\omega_{\alpha}\right\}_{i-1}\right), \\
i=\overline{1, k(\alpha)}, \alpha \in X^{0} .
\end{gathered}
$$

It is evident that between $f_{i}^{\alpha}\left(\left\{\omega_{\alpha}\right\}_{i}\right)$ there exists the following relations

$$
f_{i}^{\alpha}\left(\left\{\omega_{\alpha}\right\}_{i}\right)=\int_{\Omega_{i+1}} f_{i+1}^{\alpha}\left(\left\{\omega_{\alpha}\right\}_{i+1}\right) F_{i+1}^{\alpha}\left(\mathrm{d} \omega_{i+1}^{\alpha} \mid\left\{\omega_{\alpha}\right\}_{i}\right), i=\overline{1, k(\alpha)}, \alpha \in X^{0} .
$$

The proof of the lemma 6 is completed.

Lemma 7. Let a measurable mapping $\zeta_{t}\left(\left\{\alpha, \omega_{\alpha}\right\}\right)$ on the measurable space $\left\{\Omega, \mathcal{F}^{0}\right\}$ belong to the subclass $K^{0}$, for every fixed $\alpha \in X^{0}, i=\overline{1, k(\alpha)}$,

$$
\psi_{i}^{\alpha}\left(\left\{\omega_{\alpha}\right\}_{i-1}, a_{i}^{\alpha}\right)=f_{i-1}^{\alpha}\left(\left\{\omega_{\alpha}\right\}_{i-1}\right), \quad\left\{\omega_{\alpha}\right\}_{i-1} \in \Omega^{i-1}
$$

and there exists a constant $A<\infty$ such that

$$
\int_{X^{0}} \psi_{1}^{\alpha}(a) \mathrm{d} \mu(\alpha) \leqslant A
$$


for a certain probability measure $\mu$ on $\Sigma$. If $f_{i}^{\alpha}\left(\left\{\omega_{\alpha}\right\}_{i}\right) \geqslant 0$, then on the measurable space $\left\{\Omega, \mathcal{F}^{0}\right\}$ there exist a measure $P$ on the $\sigma$-algebra $\mathcal{F}^{0}$ and a modification $\bar{\zeta}_{t}\left(\left\{\alpha, \omega_{\alpha}\right\}\right)$ of the measurable mapping $\zeta_{t}\left(\left\{\alpha, \omega_{\alpha}\right\}\right)$, such that $\bar{\zeta}_{t}\left(\left\{\alpha, \omega_{\alpha}\right\}\right)$ is a local martingale on the probability space $\{\Omega, \mathcal{F}, P\}$ with respect to the flow of the $\sigma$-algebra $\mathcal{F}_{t}$, where the $\sigma$-algebras $\mathcal{F}$ and $\mathcal{F}_{t}$ are the completion of the $\sigma$-algebras $\mathcal{F}^{0}$ and $\mathcal{F}_{t}^{0}$ correspondingly with respect to the measure $P$.

Proof. The proof of the lemma 7 follows from the theorem 1. Really, all conditions of the theorem 1 are valid. Therefore, there exists a family of distribution functions $F_{i}^{\alpha}\left(\omega_{i}^{\alpha} \mid\left\{\omega_{\alpha}\right\}_{i-1}\right), i=\overline{1, k(\alpha)}, \alpha \in X^{0},\left\{\omega_{\alpha}\right\}_{i-1} \in \Omega^{i-1}$, with the properties, described at the introduction of the probability space $\{\Omega, \mathcal{F}, P\}$, that for $\psi_{i}^{\alpha}\left(\left\{\omega_{\alpha}\right\}_{i-1}, t\right)$ the following representation

$$
\begin{gathered}
\psi_{i}^{\alpha}\left(\left\{\omega_{\alpha}\right\}_{i-1}, t\right)=\frac{1}{1-F_{i}^{\alpha}\left(t \mid\left\{\omega_{\alpha}\right\}_{i-1}\right)} \int_{\left(t, a_{i+1}^{\alpha}\right)} f_{i}^{\alpha}\left(\left\{\omega_{\alpha}\right\}_{i}\right) F_{i}^{\alpha}\left(\mathrm{d} \omega_{i}^{\alpha} \mid\left\{\omega_{\alpha}\right\}_{i-1}\right), \\
i=\overline{1, k(\alpha)}, \alpha \in X^{0}
\end{gathered}
$$

is valid.

Let us consider the measurable mapping

$$
\begin{gathered}
\bar{\zeta}_{t}\left(\left\{\alpha, \omega_{\alpha}\right\}\right)=\sum_{i=1}^{k(\alpha)} \chi_{\left[a_{i}^{\alpha}, a_{i+1}^{\alpha}\right)}(t) \bar{\zeta}_{t}^{i, \alpha}\left(\left\{\omega_{\alpha}\right\}_{i}\right), \\
\left.+\frac{1}{1-F_{i}^{\alpha}\left(t \mid\left\{\omega_{\alpha}\right\}_{i-1}\right)} \int_{\left(t, a_{i+1}^{\alpha}\right)} f_{i}^{\alpha}\left(\left\{\omega_{\alpha}\right\}_{i}\right\}\right)=f_{i}^{\alpha}\left(\left\{\omega_{\alpha}\right\}_{i}\right) \chi_{\left.i a_{i}^{\alpha}, t\right]}^{\alpha}\left(\omega_{i}^{\alpha}\right)
\end{gathered}
$$

on the probability space $\{\Omega, \mathcal{F}, P\}$ consistent with the flow of the $\sigma$-algebras $\mathcal{F}_{t}$, where the $\sigma$-algebras $\mathcal{F}$ and $\mathcal{F}_{t}$ are the completion of the $\sigma$-algebras $\mathcal{F}^{0}$ and $\mathcal{F}_{t}^{0}$ correspondingly with respect to the measure $P$, generated by the family of distribution functions $F_{i}^{\alpha}\left(\omega_{i}^{\alpha} \mid\left\{\omega_{\alpha}\right\}_{i-1}\right), i=\overline{1, k(\alpha)}, \alpha \in X^{0}$ and the measure $\mathrm{d} \mu(\alpha)$. The measurable mapping $\zeta_{t}\left(\left\{\alpha, \omega_{\alpha}\right\}\right)$ differ from the measurable mapping $\bar{\zeta}_{t}\left(\left\{\alpha, \omega_{\alpha}\right\}\right)$ on the set $\Omega \backslash \Omega^{0}, P\left(\Omega \backslash \Omega^{0}\right)=0$. Let us construct the set $\Omega^{0}$. Consider the set $\Omega_{\alpha}^{0}=\left\{\omega_{\alpha} \in \Omega_{\alpha}, a_{i}^{\alpha}<\omega_{i}^{\alpha}<a_{i+1}^{\alpha}, i=\overline{1, k(\alpha)}\right\}$, where $\omega_{\alpha}=\left\{\omega_{1}^{\alpha}, \ldots, \omega_{k(\alpha)}^{\alpha}\right\}$ and show that $P_{\alpha}\left(\Omega_{\alpha}^{0}\right)=1$. Really, since the sequence of the sets

$$
\Omega_{\alpha}^{n}=\left\{\omega_{\alpha} \in \Omega_{\alpha}, a_{i}^{\alpha}<\omega_{i}^{\alpha}<a_{i+1}^{\alpha}, i=\overline{1, n}, a \leqslant \omega_{i}^{\alpha}<b, i=\overline{n+1, k(\alpha)}\right\}
$$

has the probability 1 , that is, $P_{\alpha}\left(\Omega_{\alpha}^{n}\right)=1, n=1,2, \ldots$, and taking into account that $\Omega_{\alpha}^{n} \supset \Omega_{\alpha}^{n+1}, \Omega_{\alpha}^{0}=\bigcap_{n=1}^{\infty} \Omega_{\alpha}^{n}$, the continuity of the probability measure $P_{\alpha}$, we obtain $P_{\alpha}\left(\Omega_{\alpha}^{0}\right)=1$. As far as there are no more than a countable set of $\alpha$ for which $\mu(\alpha)>0$, then there exists a countable subset $X_{1}^{0} \subseteq X^{0}$ such that the direct sum of the sets $\Omega_{\alpha}^{0}, \alpha \in X_{1}^{0}$ forms the set $\Omega_{0} \subset \Omega, P\left(\Omega_{0}\right)=1$. 
From the condition of the lemma 7 we have the recurrent relations

$$
\begin{aligned}
& f_{i-1}^{\alpha}\left(\left\{\omega_{\alpha}\right\}_{i-1}\right)=\int_{\left[a_{i}^{\alpha}, a_{i+1}^{\alpha}\right)} f_{i}^{\alpha}\left(\left\{\omega_{\alpha}\right\}_{i}\right) F_{i}^{\alpha}\left(\mathrm{d} \omega_{i}^{\alpha} \mid\left\{\omega_{\alpha}\right\}_{i-1}\right) \\
= & \int_{\Omega_{i}} f_{i}^{\alpha}\left(\left\{\omega_{\alpha}\right\}_{i}\right) F_{i}^{\alpha}\left(\mathrm{d} \omega_{i}^{\alpha} \mid\left\{\omega_{\alpha}\right\}_{i-1}\right), \quad i=\overline{1, k(\alpha)}, \alpha \in X^{0} .
\end{aligned}
$$

As far as

$$
\psi_{1}^{\alpha}(a)=\int_{\Omega_{1}} f_{1}^{\alpha}\left(\left\{\omega_{\alpha}\right\}_{1}\right) F_{1}^{\alpha}\left(\mathrm{d} \omega_{1}^{\alpha}\right)<\infty
$$

then we have

$$
\psi_{1}^{\alpha}(a)=\int_{\Omega_{1}} \ldots \int_{\Omega_{i}} f_{i}^{\alpha}\left(\left\{\omega_{\alpha}\right\}_{i}\right) F_{1}^{\alpha}\left(\mathrm{d} \omega_{1}^{\alpha}\right) \times \ldots \times F_{i}^{\alpha}\left(\mathrm{d} \omega_{i}^{\alpha} \mid\left\{\omega_{\alpha}\right\}_{i-1}\right) .
$$

For every $t_{0} \in[a, b)$ let us introduce the measurable mapping from the measurable space $\{\Omega, \mathcal{F}\}$ to the measurable space $\left\{R^{1}, \mathcal{B}\left(R^{1}\right)\right\}$.

$$
g_{t_{0}}\left(\left\{\alpha, \omega_{\alpha}\right\}\right)=f_{i\left(t_{0}, \alpha\right)}^{\alpha}\left(\left\{\omega_{\alpha}\right\}_{i\left(t_{0}, \alpha\right)}\right),
$$

where $i\left(t_{0}, \alpha\right)=\max \left\{i, a_{i}^{\alpha} \leqslant t_{0}\right\}$. From the condition of lemma 7

$$
M g_{t_{0}}\left(\left\{\alpha, \omega_{\alpha}\right\}\right)=\int_{X^{0}} \psi_{1}^{\alpha}(a) \mathrm{d} \mu(\alpha)<\infty .
$$

Moreover, it is not difficult to see that

$$
\bar{\zeta}_{t \wedge t_{0}}\left(\left\{\alpha, \omega_{\alpha}\right\}\right)=M\left\{g_{t_{0}}\left(\left\{\alpha, \omega_{\alpha}\right\}\right) \mid \mathcal{F}_{t}\right\} .
$$

The latter equality means that $\zeta_{t}\left(\left\{\alpha, \omega_{\alpha}\right\}\right)$ is a local martingale since this equality is valid for any $t_{0} \in[a, b)$. Therefore, we can choose the sequence of stop moments $t_{0}^{n} \rightarrow b$ with probability 1 such that $\zeta_{t \wedge t_{0}^{n}}\left(\left\{\alpha, \omega_{\alpha}\right\}\right) \rightarrow \zeta_{t}\left(\left\{\alpha, \omega_{\alpha}\right\}\right)$ with probability 1 . The lemma 7 is proved.

In a more general case, there holds

Lemma 8. Let a measurable mapping $\zeta_{t}\left(\left\{\alpha, \omega_{\alpha}\right\}\right)$ on the measurable space $\left\{\Omega, \mathcal{F}^{0}\right\}$ belong to the subclass $K^{0}$. Suppose that for any $t_{0} \in[a, b)$,

$$
\int_{X^{0}} d_{i\left(t_{0}, \alpha\right)}^{\alpha} \mathrm{d} \mu(\alpha)<\infty
$$

for a certain probability measure $\mathrm{d} \mu(\alpha)$ on $\Sigma$, where $d_{i}^{\alpha}=\sup _{\left\{\left\{\omega_{\alpha}\right\}_{i} \in \Omega^{i}\right\}}\left|f_{i}^{\alpha}\left(\left\{\omega_{\alpha}\right\}_{i}\right)\right|$,

$$
\begin{array}{r}
i\left(t_{0}, \alpha\right)=\max \left\{i, a_{i}^{\alpha} \leqslant t_{0}\right\} . \text { If for every fixed } i=\overline{1, k(\alpha)}, \alpha \in X^{0}, \\
\psi_{i}^{\alpha}\left(\left\{\omega_{\alpha}\right\}_{i-1}, a_{i}^{\alpha}\right)=f_{i}^{\alpha}\left(\left\{\omega_{\alpha}\right\}_{i-1}\right), \quad\left\{\omega_{\alpha}\right\}_{i-1} \in \Omega^{i-1},
\end{array}
$$


then on the measurable space $\left\{\Omega, \mathcal{F}^{0}\right\}$ there exist a measure $P$ on the $\sigma$-algebra $\mathcal{F}^{0}$ and a modification $\bar{\zeta}_{t}\left(\left\{\alpha, \omega_{\alpha}\right\}\right)$ of the measurable mapping $\zeta_{t}\left(\left\{\alpha, \omega_{\alpha}\right\}\right)$, such that $\bar{\zeta}_{t}\left(\left\{\alpha, \omega_{\alpha}\right\}\right)$ is a local martingale on the probability space $\{\Omega, \mathcal{F}, P\}$ with respect to the flow of the $\sigma$-algebra $\mathcal{F}_{t}$, where the $\sigma$-algebras $\mathcal{F}$ and $\mathcal{F}_{t}$ are the completion of the $\sigma$-algebras $\mathcal{F}^{0}$ and $\mathcal{F}_{t}^{0}$ correspondingly with respect to the measure $P$.

The proof of the lemma 8 is the same as the proof of the lemma 7 .

As before, let $\{\Omega, \mathcal{F}, P\}$ be the probability space with the flow of the $\sigma$-algebras $\mathcal{F}_{t} \subseteq \mathcal{F}$ on it. Suppose that $\zeta_{t}\left(\left\{\alpha, \omega_{\alpha}\right\}\right)$ is a random process consistent with the flow of $\sigma$-algebras $\mathcal{F}_{t}$, where

$$
\begin{aligned}
& \zeta_{t}\left(\left\{\alpha, \omega_{\alpha}\right\}\right)=\sum_{i=1}^{k(\alpha)} \chi_{\left[a_{i}^{\alpha}, a_{i+1}^{\alpha}\right)}(t) \zeta_{t}^{i, \alpha}\left(\left\{\omega_{\alpha}\right\}_{i}\right), \\
& \left.\zeta_{t}^{i, \alpha}\left(\left\{\omega_{\alpha}\right\}_{i}\right\}\right)=f_{i}^{\alpha}\left(\left\{\omega_{\alpha}\right\}_{i}\right) \chi_{\left[a_{i}^{\alpha}, t\right]}\left(\omega_{i}^{\alpha}\right) \\
& \quad+\frac{1}{1-F_{i}^{\alpha}\left(t \mid\left\{\omega_{\alpha}\right\}_{i-1}\right)} \int_{\left(t, a_{i+1}^{\alpha}\right)} f_{i}^{\alpha}\left(\left\{\omega_{\alpha}\right\}_{i}\right) F_{i}^{\alpha}\left(\mathrm{d} \omega_{i}^{\alpha} \mid\left\{\omega_{\alpha}\right\}_{i-1}\right) \chi_{\left(t, a_{i+1}^{\alpha}\right)}\left(\omega_{i}^{\alpha}\right),
\end{aligned}
$$

satisfying the conditions:

$$
\begin{gathered}
f_{i}^{\alpha}\left(\left\{\omega_{\alpha}\right\}_{i}\right)=\int_{\Omega_{i+1}} f_{i+1}^{\alpha}\left(\left\{\omega_{\alpha}\right\}_{i+1}\right) F_{i+1}^{\alpha}\left(\mathrm{d} \omega_{i+1}^{\alpha} \mid\left\{\omega_{\alpha}\right\}_{i}\right), \\
\int_{X^{0}} \int_{\Omega_{1}} \ldots \int_{\Omega_{i_{\alpha}}}\left|f_{i_{\alpha}}^{\alpha}\left(\left\{\alpha, \omega_{\alpha}\right\}_{i_{\alpha}}\right)\right| F_{1}^{\alpha}\left(\mathrm{d} \omega_{1}^{\alpha}\right) \ldots F_{i_{\alpha}}^{\alpha}\left(\mathrm{d} \omega_{i_{\alpha}}^{\alpha} \mid\left\{\omega_{\alpha}\right\}_{i_{\alpha}-1}\right) \mathrm{d} \mu(\alpha)<\infty
\end{gathered}
$$

for every $t_{0} \in[a, b), \quad i_{\alpha}=i\left(t_{0}, \alpha\right)=\max \left\{i, a_{i}^{\alpha} \leqslant t_{0}\right\}$, then $\zeta_{t}\left(\left\{\alpha, \omega_{\alpha}\right\}\right)$ is a local martingale. This assertion can be proved the same way as lemma 7 was proved.

Further on we connect with the local martingale $\zeta_{t}\left(\left\{\alpha, \omega_{\alpha}\right\}\right)$ on $\{\Omega, \mathcal{F}, P\}$ a stochastic process

$$
\zeta_{t}^{a}\left(\left\{\alpha, \omega_{\alpha}\right\}\right)=\sum_{i=1}^{k(\alpha)} \chi_{\left[a_{i}^{\alpha}, a_{i+1}^{\alpha}\right)}(t) \zeta_{t, a}^{i, \alpha}\left(\left\{\omega_{\alpha}\right\}_{i}\right),
$$

which is consistent with the flow of the $\sigma$-algebras $\mathcal{F}_{t}$, where

$$
\begin{gathered}
\zeta_{t, a}^{i, \alpha}\left(\left\{\omega_{\alpha}\right\}_{i}\right)= \\
=f_{i}^{\alpha}\left(\left\{\omega_{\alpha}\right\}_{i-1}, t\right)-\frac{1}{1-F_{i}^{\alpha}\left(t \mid\left\{\omega_{\alpha}\right\}_{i-1}\right)} \int_{\left(t, a_{i+1}^{\alpha}\right)} f_{i}^{\alpha}\left(\left\{\omega_{\alpha}\right\}_{i}\right) F_{i}^{\alpha}\left(\mathrm{d} \omega_{i}^{\alpha} \mid\left\{\omega_{\alpha}\right\}_{i-1}\right) .
\end{gathered}
$$

We shall call the process $\zeta_{t}^{a}\left(\left\{\alpha, \omega_{\alpha}\right\}\right)$ as the process associated with the $\zeta_{t}\left(\left\{\alpha, \omega_{\alpha}\right\}\right)$ process. 
Definition 3. Realization of the assotiated process $\zeta_{t}^{a}\left(\left\{\alpha, \omega_{\alpha}\right\}\right)$ is regular if the set

$$
\left\{t \in[a, b), \quad \zeta_{t, a}^{i, \alpha}\left(\left\{\omega_{\alpha}\right\}_{i}\right)=0, \quad i=\overline{1, k(\alpha)}\right\}
$$

is no more than the countable set.

Definition 4. A local martingal $\zeta_{t}\left(\left\{\alpha, \omega_{\alpha}\right\}\right)$ is non-singular on $\{\Omega, \mathcal{F}, P\}$ if the set of regular realizations of the associated random process $\zeta_{t}^{a}\left(\left\{\alpha, \omega_{\alpha}\right\}\right)$ has got the probability 1.

Lemma 9. On the probability space $\{\Omega, \mathcal{F}, P\}$ there always exists a non-singular local martingale.

Proof. To prove the lemma 9 we construct an example of a martingale on $\{\Omega, \mathcal{F}, P\}$ that is non-singular. Let $f_{s}^{\alpha}\left(\omega_{s}^{\alpha}\right) \geqslant 0, s=\overline{1, k(\alpha)}, \alpha \in X^{0}$ be the measurable mapping with respect to the $\sigma$-algebra $\mathcal{F}_{s}^{0}$, satisfying conditions:

$$
\begin{aligned}
& 0<\int_{\Omega_{s}} f_{s}^{\alpha}\left(\omega_{s}^{\alpha}\right) F_{s}^{\alpha}\left(\mathrm{d} \omega_{s}^{\alpha} \mid\left\{\omega_{\alpha}\right\}_{s-1}\right)<\infty \\
& \quad s=\overline{1, k(\alpha)}, \quad \alpha \in X^{0}, \quad\left\{\omega_{\alpha}\right\}_{s-1} \in \Omega^{s-1} \\
& f_{s}^{\alpha}(t)-\frac{1}{1-F_{s}^{\alpha}\left(t \mid\left\{\omega_{\alpha}\right\}_{s-1}\right)} \int_{\left(t, a_{i+1}^{\alpha}\right)} f_{s}^{\alpha}\left(\omega_{s}^{\alpha}\right) F_{s}^{\alpha}\left(\mathrm{d} \omega_{s}^{\alpha} \mid\left\{\omega_{\alpha}\right\}_{s-1}\right) \neq 0 \\
& \quad t \in\left[a_{s}^{\alpha}, a_{s+1}^{\alpha}\right), \quad s=\overline{1, k(\alpha)}, \quad \alpha \in X^{0}, \quad\left\{\omega_{\alpha}\right\}_{s-1} \in \Omega^{s-1} .
\end{aligned}
$$

Then the local martingale

$$
\xi_{t}^{\alpha}\left(\left\{\alpha, \omega_{\alpha}\right\}\right)=\sum_{i=1}^{k(\alpha)} \chi_{\left[a_{i}^{\alpha}, a_{i+1}^{\alpha}\right)}(t) \xi_{t}^{i, \alpha}\left(\left\{\omega_{\alpha}\right\}_{i}\right)
$$

is not singular, where

$$
\begin{gathered}
\left.\xi_{t}^{i, \alpha}\left(\left\{\omega_{\alpha}\right\}_{i}\right\}\right)=g_{i}^{\alpha}\left(\left\{\omega_{\alpha}\right\}_{i}\right) \chi_{\left[a_{i}^{\alpha}, t\right]}\left(\omega_{i}^{\alpha}\right) \\
+\frac{1}{1-F_{i}^{\alpha}\left(t \mid\left\{\omega_{\alpha}\right\}_{i-1}\right)} \int_{\left(t, a_{i+1}^{\alpha}\right)} g_{i}^{\alpha}\left(\left\{\omega_{\alpha}\right\}_{i}\right) F_{i}^{\alpha}\left(\mathrm{d} \omega_{i}^{\alpha} \mid\left\{\omega_{\alpha}\right\}_{i-1}\right) \chi_{\left(t, a_{i+1}^{\alpha}\right)}\left(\omega_{i}^{\alpha}\right), \\
g_{i}^{\alpha}\left(\left\{\omega_{\alpha}\right\}_{i}\right)=\prod_{s=1}^{i} g_{s}^{0, \alpha}\left(\left\{\omega_{\alpha}\right\}_{s}\right), \quad g_{s}^{0, \alpha}\left(\left\{\omega_{\alpha}\right\}_{s}\right)=\frac{f_{s}^{\alpha}\left(\omega_{s}^{\alpha}\right)}{\int_{\Omega_{s}} f_{s}^{\alpha}\left(\omega_{s}^{\alpha}\right) F_{s}^{\alpha}\left(d \omega_{s}^{\alpha} \mid\left\{\omega_{\alpha}\right\}_{s-1}\right)} .
\end{gathered}
$$

If, for example, $f_{s}^{\alpha}\left(\omega_{s}^{\alpha}\right)>0, s=\overline{1, k(\alpha)}, \alpha \in X^{0}$, are strictly monotonous on $\left[a_{s}^{\alpha}, a_{s+1}^{\alpha}\right)$, then the conditions (17), (18) are satisfied. The lemma 9 is proved. 
Theorem 3. For any local martingale $\zeta_{t}\left(\left\{\alpha, \omega_{\alpha}\right\}\right)$ given by the formula (16) and satisfying conditions $\sup _{\left\{\omega_{\alpha}\right\}_{i} \in \Omega^{i}}\left|f_{i}^{\alpha}\left(\left\{\omega_{\alpha}\right\}_{i}\right)\right|=\beta_{i}^{\alpha}<\infty, i=\overline{1, k(\alpha)}, \alpha \in X^{0}$,

$$
\int_{\left[a_{i}^{\alpha}, a_{i+1}^{\alpha}\right)}\left|\varphi_{i}^{\alpha}\left(s \mid\left\{\omega_{\alpha}\right\}_{i-1}\right)\right| \frac{F_{i}^{\alpha}\left(\mathrm{d} s \mid\left\{\omega_{\alpha}\right\}_{i-1}\right)}{1-F_{i}^{\alpha}\left(s_{-} \mid\left\{\omega_{\alpha}\right\}_{i-1}\right)}<\infty,\left\{\omega_{\alpha}\right\}_{i-1} \in \Omega^{i-1}, \alpha \in X^{0}
$$

the following representation

$$
\zeta_{t}\left(\left\{\alpha, \omega_{\alpha}\right\}\right)=\int_{\Omega_{1}} f_{1}^{\alpha}\left(\omega_{1}^{\alpha}\right) F_{1}^{\alpha}\left(\mathrm{d} \omega_{1}^{\alpha}\right)+\int_{[a, t]} \psi_{k(\alpha)}\left(s \mid \omega_{\alpha}\right) \mathrm{d} \xi_{s}\left(\left\{\alpha, \omega_{\alpha}\right\}\right), t \in[a, b)
$$

is valid if the local martingale $\xi_{t}\left(\left\{\alpha, \omega_{\alpha}\right\}\right)$ is non-singular, $\sup _{\left\{\omega_{\alpha}\right\}_{i}}\left|g_{i}^{\alpha}\left(\left\{\omega_{\alpha}\right\}_{i}\right)\right|=$ $=\delta_{i}^{\alpha}<\infty, i=\overline{1, k(\alpha)}, \alpha \in X^{0}$, and

$$
\int_{\left[a_{i}^{\alpha}, a_{i+1}^{\alpha}\right)}\left|\varphi_{i}^{0, \alpha}\left(s \mid\left\{\omega_{\alpha}\right\}_{i-1}\right)\right| \frac{F_{i}^{\alpha}\left(\mathrm{d} s \mid\left\{\omega_{\alpha}\right\}_{i-1}\right)}{1-F_{i}^{\alpha}\left(s_{-} \mid\left\{\omega_{\alpha}\right\}_{i-1}\right)}<\infty,\left\{\omega_{\alpha}\right\}_{i-1} \in \Omega^{i-1}, \alpha \in X^{0}
$$

where

$$
\begin{aligned}
& \xi_{t}\left(\left\{\alpha, \omega_{\alpha}\right\}\right)=\sum_{i=1}^{k(\alpha)} \chi_{\left[a_{i}^{\alpha}, a_{i+1}^{\alpha}\right)}(t) \xi_{t}^{i, \alpha}\left(\left\{\omega_{\alpha}\right\}_{i}\right) \\
& \left.\xi_{t}^{i, \alpha}\left(\left\{\omega_{\alpha}\right\}_{i}\right\}\right)=g_{i}^{\alpha}\left(\left\{\omega_{\alpha}\right\}_{i}\right) \chi_{\left[a_{i}^{\alpha}, t\right]}\left(\omega_{i}^{\alpha}\right) \\
& +\frac{1}{1-F_{i}^{\alpha}\left(t \mid\left\{\omega_{\alpha}\right\}_{i-1}\right)} \int_{\left(t, a_{i+1}^{\alpha}\right)} g_{i}^{\alpha}\left(\left\{\omega_{\alpha}\right\}_{i}\right) F_{i}^{\alpha}\left(\mathrm{d} \omega_{i}^{\alpha} \mid\left\{\omega_{\alpha}\right\}_{i-1}\right) \chi_{\left(t, a_{i+1}^{\alpha}\right)}\left(\omega_{i}^{\alpha}\right) \\
& \psi_{k(\alpha)}\left(s \mid \omega_{\alpha}\right)=\sum_{i=1}^{k(\alpha)} \chi_{\left[a_{i}^{\alpha}, a_{i+1}^{\alpha}\right)}(s) \frac{\varphi_{i}^{\alpha}\left(s \mid\left\{\omega_{\alpha}\right\}_{i-1}\right)}{\varphi_{i}^{0, \alpha}\left(s \mid\left\{\omega_{\alpha}\right\}_{i-1}\right)} \\
& \varphi_{i}^{0, \alpha}\left(s \mid\left\{\omega_{\alpha}\right\}_{i-1}\right)= \\
& =g_{i}^{\alpha}\left(\left\{\omega_{\alpha}\right\}_{i-1}, s\right)-\frac{1}{1-F_{i}^{\alpha}\left(s \mid\left\{\omega_{\alpha}\right\}_{i-1}\right)} \int_{\left(s, a_{i+1}^{\alpha}\right)} g_{i}^{\alpha}\left(\left\{\omega_{\alpha}\right\}_{i}\right) F_{i}^{\alpha}\left(\mathrm{d} \omega_{i}^{\alpha} \mid\left\{\omega_{\alpha}\right\}_{i-1}\right), \\
& \varphi_{i}^{\alpha}\left(s \mid\left\{\omega_{\alpha}\right\}_{i-1}\right)= \\
& =f_{i}^{\alpha}\left(\left\{\omega_{\alpha}\right\}_{i-1}, s\right)-\frac{1}{1-F_{i}^{\alpha}\left(s \mid\left\{\omega_{\alpha}\right\}_{i-1}\right)} \int_{\left(s, a_{i+1}^{\alpha}\right)} f_{i}^{\alpha}\left(\left\{\omega_{\alpha}\right\}_{i}\right) F_{i}^{\alpha}\left(\mathrm{d} \omega_{i}^{\alpha} \mid\left\{\omega_{\alpha}\right\}_{i-1}\right) .
\end{aligned}
$$


Proof. Let us consider on the right continuous version of the random processes

$$
\begin{gathered}
\xi_{t}^{1}\left(\left\{\alpha, \omega_{\alpha}\right\}\right)=\sum_{i=1}^{k(\alpha)} \chi_{\left[a_{i}^{\alpha}, a_{i+1}^{\alpha}\right)}(t) \xi_{t}^{i, \alpha, 1}\left(\left\{\omega_{\alpha}\right\}_{i}\right), \\
=\frac{1}{1-F_{i}^{\alpha}\left(\omega_{i}^{\alpha} \mid\left\{\omega_{\alpha}\right\}_{i-1}\right)} \int_{\left(\omega_{i}^{\alpha}, a_{i+1}^{\alpha}\right)} g_{i}^{\alpha}\left(\left\{\omega_{\alpha}\right\}_{i}\right) F_{i}^{\alpha}\left(\mathrm{d} \omega_{i}^{\alpha} \mid\left\{\omega_{\alpha}\right\}_{i-1}\right) \chi_{\left[a_{i}^{\alpha}, t\right)}\left(\omega_{i}^{\alpha}\right) \\
+\frac{1}{1-F_{i}^{\alpha}\left(t \mid\left\{\omega_{\alpha}\right\}_{i-1}\right)} \int_{\left(t, a_{i+1}^{\alpha}\right)} g_{i}^{\alpha}\left(\left\{\omega_{\alpha}\right\}_{i}\right) F_{i}^{\alpha}\left(\mathrm{d} \omega_{i}^{\alpha} \mid\left\{\omega_{\alpha}\right\}_{i-1}\right) \chi_{\left(t, a_{i+1}^{\alpha}\right)}\left(\omega_{i}^{\alpha}\right), \\
\xi_{t}^{2}\left(\left\{\alpha, \omega_{\alpha}\right\}\right)=\sum_{i=1}^{k(\alpha)} \chi_{\left[a_{i}^{\alpha}, a_{i+1}^{\alpha}\right)}(t) \xi_{t}^{i, \alpha, 2}\left(\left\{\omega_{\alpha}\right\}_{i}\right), \\
\xi_{t}^{i, \alpha, 2}\left(\left\{\omega_{\alpha}\right\}_{i}\right)=\chi_{\left[a_{i}^{\alpha}, t\right]}\left(\omega_{i}^{\alpha}\right) \\
1 \\
{\left[\begin{array}{c}
\left.g_{i}^{\alpha}\left(\left\{\omega_{\alpha}\right\}_{i}\right)-\frac{F_{i}\left(\omega_{i}^{\alpha} \mid\left\{\omega_{\alpha}\right\}_{i-1}\right)}{1-\omega_{\left(\omega_{i}^{\alpha}, a_{i+1}^{\alpha}\right)}} g_{i}^{\alpha}\left(\left\{\omega_{\alpha}\right\}_{i}\right) F_{i}^{\alpha}\left(\mathrm{d} \omega_{i}^{\alpha} \mid\left\{\omega_{\alpha}\right\}_{i-1}\right)\right]
\end{array}\right]}
\end{gathered}
$$

It is obvious that

$$
\xi_{t}\left(\left\{\alpha, \omega_{\alpha}\right\}\right)=\xi_{t}^{1}\left(\left\{\alpha, \omega_{\alpha}\right\}\right)+\xi_{t}^{2}\left(\left\{\alpha, \omega_{\alpha}\right\}\right) .
$$

All realizations of the random processes $\xi_{t}^{i}\left(\left\{\alpha, \omega_{\alpha}\right\}\right), i=1,2$, have got a bounded variation on any interval $[a, t], t<b$. Denote by $\mathrm{d} \xi_{t}^{i}\left(\left\{\alpha, \omega_{\alpha}\right\}\right), i=1,2$ and $\mathrm{d} \xi_{t}\left(\left\{\alpha, \omega_{\alpha}\right\}\right)$ the charges generated by these realizations on the $\sigma$-algebra $\mathcal{B}([a, b))$. To prove the theorem 3 , consider those realizations that are continuous at the points $\left\{a_{i}^{\alpha}\right\}_{i=1}^{k(\alpha)+1}, \alpha \in X^{0}$. The set of realizations satisfying this condition have got the probability 1 . The left and right limits at every point $a_{i}^{\alpha}, i=\overline{1, k(\alpha)}, \alpha \in X^{0}$ equal

$$
\begin{gathered}
\lim _{t \downarrow a_{i}^{\alpha}} \xi_{t}\left(\left\{\alpha, \omega_{\alpha}\right\}\right)=\xi_{a_{i}^{\alpha}}^{i, \alpha}\left(\left\{\omega_{\alpha}\right\}_{i}\right) \\
=\left\{\begin{array}{ccc}
\int_{\left[a_{i}^{\alpha}, a_{i+1}^{\alpha}\right)} g_{i}^{\alpha}\left(\left\{\omega_{\alpha}\right\}_{i}\right) F_{i}^{\alpha}\left(\mathrm{d} \omega_{i}^{\alpha} \mid\left\{\omega_{\alpha}\right\}_{i-1}\right), & a_{i}^{\alpha}<\omega_{i}^{\alpha}<a_{i+1}^{\alpha}, & \left\{\omega_{\alpha}\right\}_{i-1} \in \Omega^{i-1}, \\
g_{i}^{\alpha}\left(\left\{\omega_{\alpha}\right\}_{i-1}, a_{i}^{\alpha}\right), & \omega_{i}^{\alpha}=a_{i}^{\alpha}, & \left\{\omega_{\alpha}\right\}_{i-1} \in \Omega^{i-1},
\end{array}\right.
\end{gathered}
$$

$$
\lim _{t \uparrow a_{i}^{\alpha}} \xi_{t}\left(\left\{\alpha, \omega_{\alpha}\right\}\right)=g_{i-1}^{\alpha}\left(\left\{\omega_{\alpha}\right\}_{i-1}\right), \quad\left\{\omega_{\alpha}\right\}_{i-1} \in \Omega^{i-1} .
$$

Consider the set $\Omega_{\alpha}^{0}=\left\{\omega_{\alpha} \in \Omega_{\alpha}, a_{i}^{\alpha}<\omega_{i}^{\alpha}<a_{i+1}^{\alpha}, i=\overline{1, k(\alpha)}\right\}$, where $\omega_{\alpha}=$ $\left\{\omega_{1}^{\alpha}, \ldots, \omega_{k(\alpha)}^{\alpha}\right\}$ and show that $P_{\alpha}\left(\Omega_{\alpha}^{0}\right)=1$. Really, since the sequence of the sets

$$
\Omega_{\alpha}^{n}=\left\{\omega_{\alpha} \in \Omega_{\alpha}, a_{i}^{\alpha}<\omega_{i}^{\alpha}<a_{i+1}^{\alpha}, i=\overline{1, n}, a \leqslant \omega_{i}^{\alpha}<b, i=\overline{n+1, k(\alpha)}\right\}
$$


has got the probability 1 , that is, $P_{\alpha}\left(\Omega_{\alpha}^{n}\right)=1, n=1,2, \ldots$, and taking into account that $\Omega_{\alpha}^{n} \supset \Omega_{\alpha}^{n+1}, \Omega_{\alpha}^{0}=\bigcap_{n=1}^{\infty} \Omega_{\alpha}^{n}$, the continuity of the probability measure $P_{\alpha}$, we obtain $P_{\alpha}\left(\Omega_{\alpha}^{0}\right)=1$. As far as it is no more than a countable set of $\alpha$ for which $\mu(\alpha)>0$, then there exists a countable subset $X_{1}^{0} \subseteq X^{0}$ such that the direct sum of the sets $\Omega_{\alpha}^{0}, \alpha \in X_{1}^{0}$ forms the set $\Omega_{0} \subset \Omega, P\left(\Omega_{0}\right)=1$. Since

$$
\begin{aligned}
& g_{i-1}^{\alpha}\left(\left\{\omega_{\alpha}\right\}_{i-1}\right)=\int_{\left[a_{i}^{\alpha}, a_{i+1}^{\alpha}\right)} g_{i}^{\alpha}\left(\left\{\omega_{\alpha}\right\}_{i}\right) F_{i}^{\alpha}\left(\mathrm{d} \omega_{i}^{\alpha} \mid\left\{\omega_{\alpha}\right\}_{i-1}\right)= \\
& =\int_{\Omega_{i}} g_{i}^{\alpha}\left(\left\{\omega_{\alpha}\right\}_{i}\right) F_{i}^{\alpha}\left(\mathrm{d} \omega_{i}^{\alpha} \mid\left\{\omega_{\alpha}\right\}_{i-1}\right), i=\overline{1, k(\alpha)}, \alpha \in X^{0},
\end{aligned}
$$

then for every $\left\{\alpha, \omega_{\alpha}\right\} \in \Omega_{0}$ the realization of a random process $\xi_{t}\left(\left\{\alpha, \omega_{\alpha}\right\}\right)$ is continuous at the points $\left\{a_{i}^{\alpha}\right\}_{i=1}^{k(\alpha)+1}$. The charge generated by realizations of the random process $\xi_{t}^{1}\left(\left\{\alpha, \omega_{\alpha}\right\}\right)$ on the interval $\left[a_{i}^{\alpha}, a_{i+1}^{\alpha}\right)$ is absolutely continuous with respect to the measure $F_{i}^{\alpha}\left(\mathrm{d} t \mid\left\{\omega_{\alpha}\right\}_{i-1}\right)$ and the Radon-Nicodym derivative equals

$$
\begin{gathered}
\frac{\mathrm{d} \xi_{t}^{i, \alpha, 1}\left(\left\{\alpha, \omega_{\alpha}\right\}\right)}{F_{i}^{\alpha}\left(\mathrm{d} t \mid\left\{\omega_{\alpha}\right\}_{i-1}\right)}=\chi_{\left[a_{i}^{\alpha}, \omega_{i}^{\alpha}\right]}(t) \frac{1}{1-F_{i}^{\alpha}\left(t_{-} \mid\left\{\omega_{\alpha}\right\}_{i-1}\right)} \\
\times\left[\frac{1}{1-F_{i}^{\alpha}\left(t \mid\left\{\omega_{\alpha}\right\}_{i-1}\right)} \int_{\left(t, a_{i+1}^{\alpha}\right)} g_{i}^{\alpha}\left(\left\{\omega_{\alpha}\right\}_{i}\right) F_{i}^{\alpha}\left(\mathrm{d} \omega_{i}^{\alpha} \mid\left\{\omega_{\alpha}\right\}_{i-1}\right)-g_{i}^{\alpha}\left(\left\{\omega_{\alpha}\right\}_{i-1}, t\right)\right]
\end{gathered}
$$

where

$$
\frac{1}{1-F_{i}^{\alpha}\left(t_{-} \mid\left\{\omega_{\alpha}\right\}_{i-1}\right)}=\lim _{\tau \uparrow t} \frac{1}{1-F_{i}^{\alpha}\left(\tau \mid\left\{\omega_{\alpha}\right\}_{i-1}\right)} .
$$

The charge $\mathrm{d} \xi_{t}^{2}\left(\left\{\alpha, \omega_{\alpha}\right\}\right)$ generated by realizations of the process $\xi_{t}^{2}\left(\left\{\alpha, \omega_{\alpha}\right\}\right)$ on the interval $\left[a_{i}^{\alpha}, a_{i+1}^{\alpha}\right)$ is concentrated at the point $t=\omega_{i}^{\alpha}$

$$
\mathrm{d} \xi_{t}^{2}\left(\left\{\alpha, \omega_{\alpha}\right\}\right)=\mathrm{d} \xi_{t}^{i, \alpha, 2}\left(\left\{\omega_{\alpha}\right\}_{i}\right)=\delta\left(t-\omega_{i}^{\alpha}\right) \varphi_{i}^{0, \alpha}\left(\omega_{i}^{\alpha} \mid\left\{\omega_{\alpha}\right\}_{i-1}\right) .
$$

Let us calculate

$$
\begin{aligned}
& \int_{\left[a_{i}^{\alpha}, t\right]} \frac{\varphi_{i}^{\alpha}\left(s \mid\left\{\omega_{\alpha}\right\}_{i-1}\right)}{\varphi_{i}^{0, \alpha}\left(s \mid\left\{\omega_{\alpha}\right\}_{i-1}\right)} \mathrm{d} \xi_{s}^{i, \alpha}\left(\left\{\omega_{\alpha}\right\}_{i}\right)=\int_{\left[a_{i}^{\alpha}, t\right]} \frac{\varphi_{i}^{\alpha}\left(s \mid\left\{\omega_{\alpha}\right\}_{i-1}\right)}{\varphi_{i}^{0, \alpha}\left(s \mid\left\{\omega_{\alpha}\right\}_{i-1}\right)} \mathrm{d} \xi_{s}^{i, \alpha, 1}\left(\left\{\omega_{\alpha}\right\}_{i}\right) \\
& +\int_{\left[a_{i}^{\alpha}, t\right]} \frac{\varphi_{i}^{\alpha}\left(s \mid\left\{\omega_{\alpha}\right\}_{i-1}\right)}{\varphi_{i}^{0, \alpha}\left(s \mid\left\{\omega_{\alpha}\right\}_{i-1}\right)} \mathrm{d} \xi_{s}^{i, \alpha, 2}\left(\left\{\omega_{\alpha}\right\}_{i}\right)=K_{t}^{i, \alpha, 1}\left(\left\{\omega_{\alpha}\right\}_{i}\right)+K_{t}^{i, \alpha, 2}\left(\left\{\omega_{\alpha}\right\}_{i}\right) .
\end{aligned}
$$

Using (21) and the theorem 2 we have

$$
K_{t}^{i, \alpha, 1}\left(\left\{\omega_{\alpha}\right\}_{i}\right)=-\int_{\left[a_{i}^{\alpha}, t\right]} \chi_{\left[a_{i}^{\alpha}, \omega_{i}^{\alpha}\right]}(s) \varphi_{i}^{\alpha}\left(s \mid\left\{\omega_{\alpha}\right\}_{i-1}\right) \frac{F_{i}^{\alpha}\left(\mathrm{d} s \mid\left\{\omega_{\alpha}\right\}_{i-1}\right)}{1-F_{i}^{\alpha}\left(s_{-} \mid\left\{\omega_{\alpha}\right\}_{i-1}\right)}
$$




$$
\begin{gathered}
=\frac{1}{1-F_{i}^{\alpha}\left(\omega_{i}^{\alpha} \mid\left\{\omega_{\alpha}\right\}_{i-1}\right)} \int_{\left(\omega_{i}^{\alpha}, a_{i+1}^{\alpha}\right)} f_{i}^{\alpha}\left(\left\{\omega_{\alpha}\right\}_{i}\right) F_{i}^{\alpha}\left(\mathrm{d} \omega_{i}^{\alpha} \mid\left\{\omega_{\alpha}\right\}_{i-1}\right) \chi_{\left[a_{i}^{\alpha}, t\right)}\left(\omega_{i}^{\alpha}\right) \\
+\frac{1}{1-F_{i}^{\alpha}\left(t \mid\left\{\omega_{\alpha}\right\}_{i-1}\right)} \int_{\left(t, a_{i+1}^{\alpha}\right)} f_{i}^{\alpha}\left(\left\{\omega_{\alpha}\right\}_{i}\right) F_{i}^{\alpha}\left(\mathrm{d} \omega_{i}^{\alpha} \mid\left\{\omega_{\alpha}\right\}_{i-1}\right) \chi_{\left(t, a_{i+1}^{\alpha}\right)}\left(\omega_{i}^{\alpha}\right) \\
-\int_{\left(a_{i}^{\alpha}, a_{i+1}^{\alpha}\right)} f_{i}^{\alpha}\left(\left\{\omega_{\alpha}\right\}_{i}\right) F_{i}^{\alpha}\left(\mathrm{d} \omega_{i}^{\alpha} \mid\left\{\omega_{\alpha}\right\}_{i-1}\right) .
\end{gathered}
$$

Further,

$$
\times \begin{gathered}
K_{t}^{i, \alpha, 2}\left(\left\{\omega_{\alpha}\right\}_{i}\right)=\chi_{\left[a_{i}^{\alpha}, t\right]}\left(\omega_{i}^{\alpha}\right) \\
\times\left[f_{i}^{\alpha}\left(\left\{\omega_{\alpha}\right\}_{i}\right)-\frac{1}{1-F_{i}^{\alpha}\left(\omega_{i}^{\alpha} \mid\left\{\omega_{\alpha}\right\}_{i-1}\right)} \int_{\left(\omega_{i}^{\alpha}, a_{i+1}^{\alpha}\right)} f_{i}^{\alpha}\left(\left\{\omega_{\alpha}\right\}_{i}\right) F_{i}^{\alpha}\left(\mathrm{d} \omega_{i}^{\alpha} \mid\left\{\omega_{\alpha}\right\}_{i-1}\right)\right] .
\end{gathered}
$$

Therefore,

$$
\begin{gathered}
\int_{\left[a_{i}^{\alpha}, t\right]} \frac{\varphi_{i}^{\alpha}\left(s \mid\left\{\omega_{\alpha}\right\}_{i-1}\right)}{\varphi_{i}^{0, \alpha}\left(s \mid\left\{\omega_{\alpha}\right\}_{i-1}\right)} \mathrm{d} \xi_{s}^{i, \alpha}\left(\left\{\omega_{\alpha}\right\}_{i}\right)= \\
=f_{i}^{\alpha}\left(\left\{\omega_{\alpha}\right\}_{i}\right) \chi_{\left[a_{i}^{\alpha}, t\right]}\left(\omega_{i}^{\alpha}\right) \\
+\chi_{\left(t, a_{i+1}^{\alpha}\right)}\left(\omega_{i}^{\alpha}\right) \frac{1}{1-F_{i}^{\alpha}\left(t \mid\left\{\omega_{\alpha}\right\}_{i-1}\right)} \int_{\left(t, a_{i+1}^{\alpha}\right)} f_{i}^{\alpha}\left(\left\{\omega_{\alpha}\right\}_{i}\right) F_{i}^{\alpha}\left(\mathrm{d} \omega_{i}^{\alpha} \mid\left\{\omega_{\alpha}\right\}_{i-1}\right) \\
-\int_{\left[a_{i}^{\alpha}, a_{i+1}^{\alpha}\right)} f_{i}^{\alpha}\left(\left\{\omega_{\alpha}\right\}_{i}\right) F_{i}^{\alpha}\left(\mathrm{d} \omega_{i}^{\alpha} \mid\left\{\omega_{\alpha}\right\}_{i-1}\right) .
\end{gathered}
$$

Taking the limit $t \rightarrow a_{i+1}^{\alpha}$ we obtain

$$
\begin{gathered}
\int_{\left[a_{i}^{\alpha}, a_{i+1}^{\alpha}\right)} \frac{\varphi_{i}^{\alpha}\left(s \mid\left\{\omega_{\alpha}\right\}_{i-1}\right)}{\varphi_{i}^{0, \alpha}\left(s \mid\left\{\omega_{\alpha}\right\}_{i-1}\right)} \mathrm{d} \xi_{s}^{i, \alpha}\left(\left\{\omega_{\alpha}\right\}_{i}\right)= \\
=f_{i}^{\alpha}\left(\left\{\omega_{\alpha}\right\}_{i}\right)-f_{i-1}^{\alpha}\left(\left\{\omega_{\alpha}\right\}_{i-1}\right), \quad i=\overline{1, k(\alpha)},
\end{gathered}
$$

where

$$
f_{0}^{\alpha}\left(\left\{\omega_{\alpha}\right\}_{0}\right)=\int_{\left(a_{1}^{\alpha}, a_{2}^{\alpha}\right)} f_{1}^{\alpha}\left(\left\{\omega_{\alpha}\right\}_{1}\right) F_{1}^{\alpha}\left(\mathrm{d} \omega_{1}^{\alpha}\right)=\int_{\Omega_{1}} f_{1}^{\alpha}\left(\left\{\omega_{\alpha}\right\}_{1}\right) F_{1}^{\alpha}\left(\mathrm{d} \omega_{1}^{\alpha}\right) .
$$

Granting this and the definition of $\psi_{k(\alpha)}\left(s \mid \omega_{\alpha}\right)$ we obtain

$$
\begin{gathered}
\int_{\Omega_{1}} f_{1}^{\alpha}\left(\omega_{1}^{\alpha}\right) F_{1}^{\alpha}\left(\mathrm{d} \omega_{1}^{\alpha}\right)+\int_{[a, t]} \psi_{k(\alpha)}\left(s \mid \omega_{\alpha}\right) \mathrm{d} \xi_{s}\left(\left\{\alpha, \omega_{\alpha}\right\}\right)=\zeta_{t}^{i, \alpha}\left(\left\{\omega_{\alpha}\right\}_{i}\right) \\
=\zeta_{t}\left(\left\{\alpha, \omega_{\alpha}\right\}\right), t \in\left[a_{i}^{\alpha}, a_{i+1}^{\alpha}\right) .
\end{gathered}
$$

The theorem 3 is proved. 
Theorem 4. Let $\xi_{t}^{0}\left(\left\{\alpha, \omega_{\alpha}\right\}\right)$ be a local martingale on $\{\Omega, \mathcal{F}, P\}$,

$$
\begin{gathered}
\xi_{t}^{0}\left(\left\{\alpha, \omega_{\alpha}\right\}\right)=\sum_{i=1}^{k(\alpha)} \chi_{\left[a_{i}^{\alpha}, a_{i+1}^{\alpha}\right)}(t) \xi_{t}^{i, \alpha}\left(\left\{\omega_{\alpha}\right\}_{i}\right), \\
\left.\xi_{t}^{i, \alpha}\left(\left\{\omega_{\alpha}\right\}_{i}\right\}\right)=g_{i}^{\alpha}\left(\left\{\omega_{\alpha}\right\}_{i}\right) \chi_{\left[a_{i}^{\alpha}, t\right]}\left(\omega_{i}^{\alpha}\right) \\
+\frac{1}{1-F_{i}^{\alpha}\left(t \mid\left\{\omega_{\alpha}\right\}_{i-1}\right)} \int_{\left(t, a_{i+1}^{\alpha}\right)} g_{i}^{\alpha}\left(\left\{\omega_{\alpha}\right\}_{i}\right) F_{i}^{\alpha}\left(\mathrm{d} \omega_{i}^{\alpha} \mid\left\{\omega_{\alpha}\right\}_{i-1}\right) \chi_{\left(t, a_{i+1}^{\alpha}\right)}\left(\omega_{i}^{\alpha}\right),
\end{gathered}
$$

satisfying conditions:

$$
\begin{gathered}
\sup _{\left\{\omega_{\alpha}\right\}_{i} \in \Omega^{i}}\left|g_{i}^{\alpha}\left(\left\{\omega_{\alpha}\right\}_{i}\right)\right|=\beta_{i}^{\alpha}<\infty, i=\overline{1, k(\alpha)}, \alpha \in X^{0}, \\
\int_{\left[a_{i}^{\alpha}, a_{i+1}^{\alpha}\right)}\left|\varphi_{i}^{0, \alpha}\left(s \mid\left\{\omega_{\alpha}\right\}_{i-1}\right)\right| \frac{F_{i}^{\alpha}\left(\mathrm{d} s \mid\left\{\omega_{\alpha}\right\}_{i-1}\right)}{1-F_{i}^{\alpha}\left(s_{-} \mid\left\{\omega_{\alpha}\right\}_{i-1}\right)}<\infty, \\
\left\{\omega_{\alpha}\right\}_{i-1} \in \Omega^{i-1}, i=\overline{1, k(\alpha)}, \alpha \in X^{0},
\end{gathered}
$$

where

$$
\begin{gathered}
\varphi_{i}^{0, \alpha}\left(s \mid\left\{\omega_{\alpha}\right\}_{i-1}\right)= \\
=g_{i}^{\alpha}\left(\left\{\omega_{\alpha}\right\}_{i-1}, s\right)-\frac{1}{1-F_{i}^{\alpha}\left(s \mid\left\{\omega_{\alpha}\right\}_{i-1}\right)} \int_{\left(s, a_{i+1}^{\alpha}\right)} g_{i}^{\alpha}\left(\left\{\omega_{\alpha}\right\}_{i}\right) F_{i}^{\alpha}\left(\mathrm{d} \omega_{i}^{\alpha} \mid\left\{\omega_{\alpha}\right\}_{i-1}\right) .
\end{gathered}
$$

The random process

$$
\xi_{t}\left(\left\{\alpha, \omega_{\alpha}\right\}\right)=\sum_{i=1}^{k(\alpha)} \chi_{\left[a_{i}^{\alpha}, a_{i+1}^{\alpha}\right)}(t) f_{\alpha}\left(\xi_{t}^{i, \alpha}\left(\left\{\omega_{\alpha}\right\}_{i}\right)\right)
$$

belongs to the subclass $K_{0}$ if the family of functions $f_{\alpha}(x)>0, x \in R^{1}, \alpha \in X^{0}$, are strictly monotonous, $\sup _{x \in R^{1}}\left|f_{\alpha}^{\prime}(x)\right|=f_{1}^{\alpha}<\infty, \inf _{x \in R^{1}}\left|f_{\alpha}^{\prime}(x)\right|=f_{2}^{\alpha}>0$, moreover,

$$
\sup _{i} \sup _{\left\{\omega_{\alpha}\right\}_{i-1} \in \Omega^{i-1}} \sup _{s \in\left[a_{i}^{\alpha}, a_{i+1}^{\alpha}\right)} \frac{\Delta F_{i}^{\alpha}\left(s \mid\left\{\omega_{\alpha}\right\}_{i-1}\right)}{1-F_{i}^{\alpha}\left(s_{-} \mid\left\{\omega_{\alpha}\right\}_{i-1}\right)}<\frac{f_{2}^{\alpha}}{f_{1}^{\alpha}} .
$$

Proof. To prove the theorem 4 it is sufficient to verify the fulfillment of the conditions of definition 2. The condition 1 is valid, because

$$
\psi_{i}^{\alpha}\left(\left\{\omega_{\alpha}\right\}_{i-1}, t\right)=f_{\alpha}\left(T_{i}^{\alpha}\left(t \mid\left\{\omega_{\alpha}\right\}_{i-1}\right)\right)
$$

is continuous on the right, where

$$
T_{i}^{\alpha}\left(t \mid\left\{\omega_{\alpha}\right\}_{i-1}\right)=\frac{1}{1-F_{i}^{\alpha}\left(t \mid\left\{\omega_{\alpha}\right\}_{i-1}\right)} \int_{\left(t, a_{i+1}^{\alpha}\right)} g_{i}^{\alpha}\left(\left\{\omega_{\alpha}\right\}_{i}\right) F_{i}^{\alpha}\left(\mathrm{d} \omega_{i}^{\alpha} \mid\left\{\omega_{\alpha}\right\}_{i-1}\right) .
$$


Moreover,

$$
\begin{gathered}
\operatorname{var}_{t \in\left[a_{i}^{\alpha}, \tau\right]} \psi_{i}^{\alpha}\left(\left\{\omega_{\alpha}\right\}_{i-1}, t\right) \leqslant f_{1}^{\alpha} \int_{\left[a_{i}^{\alpha}, \tau\right]}\left|\varphi_{i}^{0, \alpha}\left(s \mid\left\{\omega_{\alpha}\right\}_{i-1}\right)\right| \frac{F_{i}^{\alpha}\left(\mathrm{d} s \mid\left\{\omega_{\alpha}\right\}_{i-1}\right)}{1-F_{i}^{\alpha}\left(s_{-} \mid\left\{\omega_{\alpha}\right\}_{i-1}\right)}<\infty \\
\tau \in\left[a_{i}^{\alpha}, a_{i+1}^{\alpha}\right), \quad\left\{\omega_{\alpha}\right\}_{i-1} \in \Omega^{i-1}, \alpha \in X^{0} .
\end{gathered}
$$

The Radon-Nicodym derivative of the charge $\psi_{i}^{\alpha}\left(\left\{\omega_{\alpha}\right\}_{i-1}, \mathrm{~d} t\right)$, generated by $\psi_{i}^{\alpha}\left(\left\{\omega_{\alpha}\right\}_{i-1}, t\right)$, equals

$$
\frac{\psi_{i}^{\alpha}\left(\left\{\omega_{\alpha}\right\}_{i-1}, \mathrm{~d} t\right)}{F_{i}^{\alpha}\left(\mathrm{d} t \mid\left\{\omega_{\alpha}\right\}_{i-1}\right)}=-\frac{f_{\alpha}^{\prime}\left(T_{i}^{\alpha}\left(t \mid\left\{\omega_{\alpha}\right\}_{i-1}\right)\right) \varphi_{i}^{0, \alpha}\left(t \mid\left\{\omega_{\alpha}\right\}_{i-1}\right)}{1-F_{i}^{\alpha}\left(t_{-} \mid\left\{\omega_{\alpha}\right\}_{i-1}\right)} .
$$

Thus,

$$
\begin{gathered}
\gamma^{i, \alpha}\left(\left\{\omega_{\alpha}\right\}_{i-1}, t\right)=\int_{\left[a_{i}^{\alpha}, t\right]} \frac{\psi_{i}^{\alpha}\left(\left\{\omega_{\alpha}\right\}_{i-1}, \mathrm{~d} \tau\right)}{\psi_{i}^{\alpha}\left(\left\{\omega_{\alpha}\right\}_{i-1}, \tau\right)-f_{\alpha}\left(g_{i}^{\alpha}\left(\left\{\omega_{\alpha}\right\}_{i-1}, \tau\right)\right)} \\
=\int_{\left[a_{i}^{\alpha}, t\right]} \frac{f_{\alpha}^{\prime}\left(T_{i}^{\alpha}\left(\tau \mid\left\{\omega_{\alpha}\right\}_{i-1}\right)\right) F_{i}^{\alpha}\left(\mathrm{d} \tau \mid\left\{\omega_{\alpha}\right\}_{i-1}\right)}{U\left(\left\{\omega_{\alpha}\right\}_{i-1}, \tau\right)\left[1-F_{i}^{\alpha}\left(\tau_{-} \mid\left\{\omega_{\alpha}\right\}_{i-1}\right)\right]}
\end{gathered}
$$

is non-negative and monotonously non-decreasing on $\left[a_{i}^{\alpha}, a_{i+1}^{\alpha}\right)$, where

$$
U\left(\left\{\omega_{\alpha}\right\}_{i-1}, \tau\right)=\int_{0}^{1} f_{\alpha}^{\prime}\left(g_{i}^{\alpha}\left(\left\{\omega_{\alpha}\right\}_{i-1}, \tau\right)+z\left[T_{i}^{\alpha}\left(\tau \mid\left\{\omega_{\alpha}\right\}_{i-1}\right)-g_{i}^{\alpha}\left(\left\{\omega_{\alpha}\right\}_{i-1}, \tau\right)\right]\right) \mathrm{d} z
$$

Further,

$$
\begin{gathered}
\Delta \gamma^{i, \alpha}\left(\left\{\omega_{\alpha}\right\}_{i-1}, t\right) \leqslant \frac{f_{1}^{\alpha}}{f_{2}^{\alpha}} \sup _{i} \sup _{\left\{\omega_{\alpha}\right\}_{i-1} \in \Omega^{i-1}} \sup _{s \in\left[a_{i}^{\alpha}, a_{i+1}^{\alpha}\right)} \frac{\Delta F_{i}^{\alpha}\left(s \mid\left\{\omega_{\alpha}\right\}_{i-1}\right)}{1-F_{i}^{\alpha}\left(s_{-} \mid\left\{\omega_{\alpha}\right\}_{i-1}\right)}<1 . \\
\lim _{t \rightarrow a_{i+1}^{\alpha}} \gamma^{i, \alpha}\left(\left\{\omega_{\alpha}\right\}_{i-1}, t\right) \geqslant \frac{f_{2}^{\alpha}}{f_{1}^{\alpha}} \lim _{t \rightarrow a_{i+1}^{\alpha}} \int_{\left[a_{i}^{\alpha}, t\right]} \frac{F_{i}^{\alpha}\left(\mathrm{d} \tau \mid\left\{\omega_{\alpha}\right\}_{i-1}\right)}{1-F_{i}^{\alpha}\left(\tau_{-} \mid\left\{\omega_{\alpha}\right\}_{i-1}\right)}=\infty \\
\lim _{t \rightarrow a_{i}^{\alpha}} \gamma^{i, \alpha}\left(\left\{\omega_{\alpha}\right\}_{i-1}, t\right)=0,\left\{\omega_{\alpha}\right\}_{i-1} \in \Omega^{i-1}, \alpha \in X^{0} .
\end{gathered}
$$

(c) is evident from (b) and boundedness of $\psi_{i}^{\alpha}\left(\left\{\omega_{\alpha}\right\}_{i}\right)$.

At last

$$
\begin{gathered}
\int_{\left[a_{i}^{\alpha}, a_{i+1}^{\alpha}\right)}\left|f_{\alpha}\left(g_{i}^{\alpha}\left(\left\{\omega_{\alpha}\right\}_{i-1}, t\right)\right)\right| \exp \left\{-\gamma^{i, \alpha}\left(\left\{\omega_{\alpha}\right\}_{i-1}, t_{-}\right)\right\} \gamma^{i, \alpha}\left(\left\{\omega_{\alpha}\right\}_{i-1}, \mathrm{~d} t\right) \leqslant \\
\leqslant \mathrm{e}\left(\beta_{i}^{\alpha} f_{1}^{\alpha}+f_{\alpha}(0)\right) .
\end{gathered}
$$

The theorem 4 is proved. 


\section{Options and their pricing}

We assume that $\{\Omega, \mathcal{F}, P\}$ is a full probability space, generated by the family of distribution functions $F_{i}^{\alpha}\left(\omega_{i}^{\alpha} \mid\left\{\omega_{\alpha}\right\}_{i-1}\right), i=\overline{1, k(\alpha)}, \alpha \in X^{0}$ and a measure $\mathrm{d} \mu(\alpha)$ on the $\sigma$-algebra $\Sigma$. Further on we assume that $X^{0}$ is a space of possible hypothesis each of which may occur with probability $\mu(\alpha)$, that is, an evolution of stock price can come by one of the possible scenario. This scenario is determined by sequence $\alpha$ and a probability space $\left\{\Omega_{\alpha}, \mathcal{F}_{\alpha}^{0}, P_{\alpha}\right\}$.

Theorem 5. Let $\phi\left(\left\{\alpha, \omega_{\alpha}\right\}\right)=\phi^{\alpha}\left(\left\{\omega_{\alpha}\right\}_{k(\alpha)}\right)$ be a random value on the probability space $\{\Omega, \mathcal{F}, P\}$, satisfying conditions:

1) $\left|\phi^{\alpha}\left(\left\{\omega_{\alpha}\right\}_{k(\alpha)}\right)\right| \leqslant C_{\alpha}<\infty, \quad \alpha \in X^{0}, \int_{X^{0}} C_{\alpha} \mathrm{d} \mu(\alpha)<\infty$;

2) there exists $t_{i}^{\alpha} \in\left[a_{i}^{\alpha}, a_{i+1}^{\alpha}\right)$ such that

$$
\begin{gathered}
\left|\phi^{\alpha}\left(\left\{\omega_{\alpha}\right\}_{i-1}, s_{1},\left\{\omega_{\alpha}\right\}_{[i+1, k(\alpha)]}\right)-\phi^{\alpha}\left(\left\{\omega_{\alpha}\right\}_{i-1}, s_{2},\left\{\omega_{\alpha}\right\}_{[i+1, k(\alpha)]}\right)\right| \leqslant \\
\leqslant C_{i}^{\alpha}\left|F_{i}^{\alpha}\left(s_{1} \mid\left\{\omega_{\alpha}\right\}_{i-1}\right)-F_{i}^{\alpha}\left(s_{2} \mid\left\{\omega_{\alpha}\right\}_{i-1}\right)\right|^{\varepsilon_{\alpha}^{i}}, \varepsilon_{\alpha}^{i}>0, s_{1}, s_{2} \in\left[t_{i}^{\alpha}, a_{i+1}^{\alpha}\right), \\
C_{i}^{\alpha}<\infty, i=\overline{1, k(\alpha)},\left\{\omega_{\alpha}\right\}_{i-1} \in \Omega^{i-1},\left\{\omega_{\alpha}\right\}_{[i+1, k(\alpha)]} \in \Omega^{k(\alpha)-i}, \alpha \in X^{0} .
\end{gathered}
$$

Further, let $\xi_{t}^{0}\left(\left\{\alpha, \omega_{\alpha}\right\}\right)$ be a local non-singular martingale on $\{\Omega, \mathcal{F}, P\}$,

$$
\begin{gathered}
\xi_{t}^{0}\left(\left\{\alpha, \omega_{\alpha}\right\}\right)=\sum_{i=1}^{k(\alpha)} \chi_{\left[a_{i}^{\alpha}, a_{i+1}^{\alpha}\right)}(t) \xi_{t}^{i, \alpha}\left(\left\{\omega_{\alpha}\right\}_{i}\right), \\
+\frac{\left.\xi_{t}^{i, \alpha}\left(\left\{\omega_{\alpha}\right\}_{i}\right\}\right)=g_{i}^{\alpha}\left(\left\{\omega_{\alpha}\right\}_{i}\right) \chi_{\left[a_{i}^{\alpha}, t\right]}\left(\omega_{i}^{\alpha}\right)}{1-F_{i}^{\alpha}\left(t \mid\left\{\omega_{\alpha}\right\}_{i-1}\right)} \int_{\left(t, a_{i+1}^{\alpha}\right)} g_{i}^{\alpha}\left(\left\{\omega_{\alpha}\right\}_{i}\right) F_{i}^{\alpha}\left(\mathrm{d} \omega_{i}^{\alpha} \mid\left\{\omega_{\alpha}\right\}_{i-1}\right) \chi_{\left(t, a_{i+1}^{\alpha}\right)}\left(\omega_{i}^{\alpha}\right),
\end{gathered}
$$

satisfying conditions

$$
\begin{gathered}
\sup _{\left\{\omega_{\alpha}\right\}_{i} \in \Omega^{i}}\left|g_{i}^{\alpha}\left(\left\{\omega_{\alpha}\right\}_{i}\right)\right|=\beta_{i}^{\alpha}<\infty, i=\overline{1, k(\alpha)}, \alpha \in X^{0}, \\
\int_{\left[a_{i}^{\alpha}, a_{i+1}^{\alpha}\right)}\left|\rho_{i}^{0, \alpha}\left(s \mid\left\{\omega_{\alpha}\right\}_{i-1}\right)\right| \frac{F_{i}^{\alpha}\left(\mathrm{d} s \mid\left\{\omega_{\alpha}\right\}_{i-1}\right)}{1-F_{i}^{\alpha}\left(s_{-} \mid\left\{\omega_{\alpha}\right\}_{i-1}\right)}<\infty,\left\{\omega_{\alpha}\right\}_{i-1} \in \Omega^{i-1}, \alpha \in X^{0}, \\
=g_{i}^{\alpha}\left(\left\{\omega_{\alpha}\right\}_{i-1}, s\right)-\frac{1}{1-F_{i}^{\alpha}\left(s \mid\left\{\omega_{\alpha}\right\}_{i-1}\right)} \int_{\left(s, a_{i+1}^{\alpha}\right)} g_{i}^{\alpha}\left(\left\{\omega_{\alpha}\right\}_{i}\right) F_{i}^{\alpha}\left(\mathrm{d} \omega_{i}^{\alpha} \mid\left\{\omega_{\alpha}\right\}_{i-1}\right),
\end{gathered}
$$

If the random process has got the form

$$
\xi_{t}\left(\left\{\alpha, \omega_{\alpha}\right\}\right)=\sum_{i=1}^{k(\alpha)} \chi_{\left[a_{i}^{\alpha}, a_{i+1}^{\alpha}\right)}(t) f_{\alpha}\left(\xi_{t}^{i, \alpha}\left(\left\{\omega_{\alpha}\right\}_{i}\right)\right),
$$


where a family of functions $f_{\alpha}(x)>0, x \in R^{1}, \alpha \in \in X^{0}$, is such that each of the functions $f_{\alpha}(x)$ is strictly fulfillment, $\sup _{x \in R^{1}}\left|f_{\alpha}^{\prime}(x)\right|=f_{1}^{\alpha}<\infty, \inf _{x \in R^{1}}\left|f_{\alpha}^{\prime}(x)\right|=f_{2}^{\alpha}>0$, and

$$
\sup _{i} \sup _{\left\{\omega_{\alpha}\right\}_{i-1} \in \Omega^{i-1}} \sup _{s \in\left[a_{i}^{\alpha}, a_{i+1}^{\alpha}\right)} \frac{\Delta F_{i}^{\alpha}\left(s \mid\left\{\omega_{\alpha}\right\}_{i-1}\right)}{1-F_{i}^{\alpha}\left(s_{-} \mid\left\{\omega_{\alpha}\right\}_{i-1}\right)}<\frac{f_{2}^{\alpha}}{f_{1}^{\alpha}}
$$

then there exists a measure $P_{1}$ on $\left\{\Omega, \mathcal{F}^{0}\right\}$, generated by a certain family of distribution functions $F_{i}^{\alpha, 1}\left(\omega_{i}^{\alpha} \mid\left\{\omega_{\alpha}\right\}_{i-1}\right), i=\overline{1, k(\alpha)}, \alpha \in X^{0}$, a probability measure $\mathrm{d} \mu_{1}(\alpha)$ on the $\sigma$-algebra $\Sigma$ and a modification $\bar{\xi}_{t}\left(\left\{\alpha, \omega_{\alpha}\right\}\right)$ of the the random process $\xi_{t}\left(\left\{\alpha, \omega_{\alpha}\right\}\right)$ such that $\bar{\xi}_{t}\left(\left\{\alpha, \omega_{\alpha}\right\}\right)$ is a local non-singular martingale on the probability space $\left\{\Omega, \mathcal{F}_{1}, P_{1}\right\}$ with respect to the flow of the $\sigma$-algebras $\mathcal{F}_{t}^{1}$, where the $\sigma$-algebras $\mathcal{F}_{1}$ and $\mathcal{F}_{t}^{1}$ are the completion of the $\sigma$-algebras $\mathcal{F}^{0}$ and $\mathcal{F}_{t}^{0}$ correspondingly with respect to the measure $P_{1}$. Moreover, for the regular martingale $M^{1}\left\{\phi\left(\left\{\alpha, \omega_{\alpha}\right\}\right) \mid \mathcal{F}_{t}^{1}\right\}$ on the probability space $\left\{\Omega, \mathcal{F}_{1}, P_{1}\right\}$ the representation

$$
M^{1}\left\{\phi\left(\left\{\alpha, \omega_{\alpha}\right\}\right) \mid \mathcal{F}_{t}\right\}=M_{\alpha}^{1} \phi^{\alpha}\left(\left\{\omega_{\alpha}\right\}_{k(\alpha)}\right)+\int_{[a, t]} \psi_{k(\alpha)}\left(s \mid \omega_{\alpha}\right) \mathrm{d} \bar{\xi}_{s}\left(\left\{\alpha, \omega_{\alpha}\right\}\right), t \in[a, b)
$$

is valid, where

$$
\begin{gathered}
\psi_{k(\alpha)}\left(s \mid \omega_{\alpha}\right)=\sum_{i=1}^{k(\alpha)} \chi_{\left[a_{i}^{\alpha}, a_{i+1}^{\alpha}\right)}(s) \frac{\varphi_{i}^{\alpha}\left(s \mid\left\{\omega_{\alpha}\right\}_{i-1}\right)}{\varphi_{i}^{0, \alpha}\left(s \mid\left\{\omega_{\alpha}\right\}_{i-1}\right)}, \\
=f_{\alpha}\left(g_{i}^{\alpha}\left(\left\{\omega_{\alpha}\right\}_{i-1}, s\right)\right)-\frac{1}{1-F_{i}^{\alpha, 1}\left(s \mid\left\{\omega_{\alpha}\right\}_{i-1}\right)} \int_{\left(s, a_{i+1}^{\alpha}\right)} f_{\alpha}\left(g_{i}^{\alpha}\left(\left\{\omega_{\alpha}\right\}_{i}\right)\right) F_{i}^{\alpha, 1}\left(\mathrm{~d} \omega_{i}^{\alpha} \mid\left\{\omega_{\alpha}\right\}_{i-1}\right), \\
=\phi_{i}^{\alpha}\left(\left\{\omega_{\alpha}\right\}_{i-1}, s\right)-\frac{1}{1-F_{i}^{\alpha, 1}\left(s \mid\left\{\omega_{\alpha}\right\}_{i-1}\right)} \int_{\left(s, a_{i+1}^{\alpha}\right)} \phi_{i}^{\alpha}\left(\left\{\omega_{\alpha}\right\}_{i}\right) F_{i}^{\alpha, 1}\left(\mathrm{~d} \omega_{i}^{\alpha} \mid\left\{\omega_{\alpha}\right\}_{i-1}\right)= \\
\phi_{i}^{\alpha}\left(\left\{\omega_{\alpha}\right\}_{i}\right)=\int_{\left.\Omega_{i+1}\right),} \int_{\Omega_{k(\alpha)}} \phi^{\alpha}\left(\left\{\omega_{\alpha}\right\}_{i},\left\{\omega_{\alpha}\right\}_{[i+1, k(\alpha)]}\right) \\
\times F_{i+1}^{\alpha, 1}\left(\mathrm{~d} \omega_{i+1}^{\alpha} \mid\left\{\omega_{\alpha}\right\}_{i}\right) \times \ldots \times F_{k(\alpha)}^{\alpha, 1}\left(\mathrm{~d} \omega_{k(\alpha)}^{\alpha} \mid\left\{\omega_{\alpha}\right\}_{k(\alpha)-1}\right) .
\end{gathered}
$$

Proof. The conditions of the theorem 5 guarantee the monotonous of the conditions of the theorem 4 . Therefore the random process $\xi_{t}\left(\left\{\alpha, \omega_{\alpha}\right\}\right)$ belongs to the subclass $K^{0}$. Moreover,

$$
\psi_{i}^{\alpha}\left(\left\{\omega_{\alpha}\right\}_{i-1}, a_{i}^{\alpha}\right)=f_{\alpha}\left(\xi_{a_{i}^{\alpha}}^{i, \alpha}\left(\left\{\omega_{\alpha}\right\}_{i}\right)\right)=f_{\alpha}\left(\int_{\left(a_{i}^{\alpha}, a_{i+1}^{\alpha}\right)} g_{i}^{\alpha}\left(\left\{\omega_{\alpha}\right\}_{i}\right) F_{i}^{\alpha}\left(\mathrm{d} \omega_{i}^{\alpha} \mid\left\{\omega_{\alpha}\right\}_{i-1}\right)\right)=
$$




$$
=f_{\alpha}\left(g_{i-1}^{\alpha}\left(\left\{\omega_{\alpha}\right\}_{i-1}\right)\right)=f_{i-1}^{\alpha}\left(\left\{\omega_{\alpha}\right\}_{i-1}\right)
$$

with probability 1 on the probability space $\{\Omega, \mathcal{F}, P\}$, since

$$
g_{i-1}^{\alpha}\left(\left\{\omega_{\alpha}\right\}_{i-1}\right)=\int_{\left(a_{i}^{\alpha}, a_{i+1}^{\alpha}\right)} g_{i}^{\alpha}\left(\left\{\omega_{\alpha}\right\}_{i}\right) F_{i}^{\alpha}\left(\mathrm{d} \omega_{i}^{\alpha} \mid\left\{\omega_{\alpha}\right\}_{i-1}\right)
$$

with probability 1. Further,

$$
\int_{X^{0}} f_{\alpha}(v(\alpha)) \mathrm{d} \mu_{1}(\alpha)<\infty
$$

where $\mathrm{d} \mu_{1}(\alpha)=u(\alpha) \mathrm{d} \mu(\alpha)$,

$$
\begin{gathered}
v(\alpha)=\int_{\left(a_{i}^{\alpha}, a_{i+1}^{\alpha}\right)} g_{1}^{\alpha}\left(\omega_{1}^{\alpha}\right) F_{1}^{\alpha}\left(\mathrm{d} \omega_{1}^{\alpha}\right), \\
u(\alpha)=\frac{\chi_{A}(\alpha)+\left[f_{\alpha}(v(\alpha))\right]^{-1} \chi_{X^{0} \backslash A}(\alpha)}{D}, \\
D=\int_{X^{0}}\left\{\chi_{A}(\alpha)+\left[f_{\alpha}(v(\alpha))\right]^{-1} \chi_{X^{0} \backslash A}(\alpha)\right\} \mathrm{d} \mu(\alpha),
\end{gathered}
$$

$\chi_{A}(\alpha)$ is the characteristic function of the set $A=\left\{\alpha, f_{\alpha}(v(\alpha)) \leqslant 1\right\}$. Based on the lemma 7 there exists a measure $P_{1}$ on the $\sigma$-algebra $\mathcal{F}^{0}$, generated by a certain family of distribution functions $F_{i}^{\alpha, 1}\left(\omega_{i}^{\alpha} \mid\left\{\omega_{\alpha}\right\}_{i-1}\right), i=\overline{1, k(\alpha)}, \alpha \in X^{0}$ and the measure $\mathrm{d} \mu_{1}(\alpha)$ on $\sigma$-algebra $\Sigma$ such that the random process

$$
\begin{gathered}
\bar{\xi}_{t}\left(\left\{\alpha, \omega_{\alpha}\right\}\right)=\sum_{i=1}^{k(\alpha)} \chi_{\left[a_{i}^{\alpha}, a_{i+1}^{\alpha}\right)}(t) \bar{\xi}_{t}^{i, \alpha}\left(\left\{\omega_{\alpha}\right\}_{i}\right), \\
+\frac{1}{1-F_{i}^{\alpha, 1}\left(t \mid\left\{\omega_{\alpha}\right\}_{i-1}\right)} \int_{\left(t, a_{i+1}^{\alpha}\right)} f_{\alpha}\left(g_{i}^{\alpha}\left(\left\{\omega_{\alpha}\right\}_{i}\right)\right) F_{i}^{\alpha, 1}\left(\mathrm{~d} \omega_{i}^{\alpha} \mid\left\{\omega_{\alpha}\right\}_{i-1}\right) \chi_{\left(t, a_{i+1}^{\alpha}\right)}\left(\omega_{i}^{\alpha}\right), \\
i=\overline{1, k(\alpha)}, \quad \alpha \in X^{0},
\end{gathered}
$$

is a modification of the random process $\xi_{t}\left(\left\{\alpha, \omega_{\alpha}\right\}\right)$ on the probability space $\left\{\Omega, \mathcal{F}_{1}, P_{1}\right\}$.

Since $\xi_{t}^{0}\left(\left\{\alpha, \omega_{\alpha}\right\}\right)$ is also a local non-singular martingale, then $\bar{\xi}_{t}\left(\left\{\alpha, \omega_{\alpha}\right\}\right)$ is also a local non-singular martingale because

$$
\begin{aligned}
& \left\{t \in[a, b), \bar{\xi}_{t, a}^{i, \alpha}\left(\left\{\omega_{\alpha}\right\}_{i-1}\right)=0, i=\overline{1, k(\alpha)}\right\} \subseteq \\
& \subseteq\left\{t \in[a, b), \xi_{t, a}^{i, \alpha}\left(\left\{\omega_{\alpha}\right\}_{i-1}\right)=0, i=\overline{1, k(\alpha)}\right\},
\end{aligned}
$$


due to the strict monotony of $f_{\alpha}(x)$, where

$$
\begin{aligned}
& \bar{\xi}_{t, a}^{i, \alpha}\left(\left\{\omega_{\alpha}\right\}_{i-1}\right)= \\
& =f_{\alpha}\left(g_{i}^{\alpha}\left(\left\{\omega_{\alpha}\right\}_{i}\right)\right)-\frac{1}{1-F_{i}^{\alpha, 1}\left(t \mid\left\{\omega_{\alpha}\right\}_{i-1}\right)} \int_{\left(t, a_{i+1}^{\alpha}\right)} f_{\alpha}\left(g_{i}^{\alpha}\left(\left\{\omega_{\alpha}\right\}_{i}\right)\right) F_{i}^{\alpha, 1}\left(\mathrm{~d} \omega_{i}^{\alpha} \mid\left\{\omega_{\alpha}\right\}_{i-1}\right) \\
& =f_{\alpha}\left(g_{i}^{\alpha}\left(\left\{\omega_{\alpha}\right\}_{i}\right)\right)-f_{\alpha}\left(T_{i}^{\alpha}\left(t \mid\left\{\omega_{\alpha}\right\}_{i-1}\right)\right), \\
& T_{i}^{\alpha}\left(t \mid\left\{\omega_{\alpha}\right\}_{i-1}\right)=\frac{1}{1-F_{i}^{\alpha}\left(t \mid\left\{\omega_{\alpha}\right\}_{i-1}\right)} \int_{\left(t, a_{i+1}^{\alpha}\right)} g_{i}^{\alpha}\left(\left\{\omega_{\alpha}\right\}_{i}\right) F_{i}^{\alpha}\left(\mathrm{d} \omega_{i}^{\alpha} \mid\left\{\omega_{\alpha}\right\}_{i-1}\right), \\
& \xi_{t, a}^{i, \alpha}\left(\left\{\omega_{\alpha}\right\}_{i-1}\right)=g_{i}^{\alpha}\left(\left\{\omega_{\alpha}\right\}_{i}\right)-T_{i}^{\alpha}\left(t \mid\left\{\omega_{\alpha}\right\}_{i-1}\right) .
\end{aligned}
$$

To finish the proof of the theorem 5 it is sufficient to verify the monotonous of the conditions of the theorem 3. Really,

$$
\begin{gathered}
\int_{\left[a_{i}^{\alpha}, a_{i+1}^{\alpha}\right)}\left|\varphi_{i}^{0, \alpha}\left(s \mid\left\{\omega_{\alpha}\right\}_{i-1}\right)\right| \frac{F_{i}^{\alpha, 1}\left(\mathrm{~d} s \mid\left\{\omega_{\alpha}\right\}_{i-1}\right)}{1-F_{i}^{\alpha, 1}\left(s_{-} \mid\left\{\omega_{\alpha}\right\}_{i-1}\right)} \leqslant \\
\leqslant \frac{\left[f_{1}^{\alpha}\right]^{2}}{f_{2}^{\alpha}} \int_{\left[a_{i}^{\alpha}, a_{i+1}^{\alpha}\right)}\left|\rho_{i}^{0, \alpha}\left(s \mid\left\{\omega_{\alpha}\right\}_{i-1}\right)\right| \frac{F_{i}^{\alpha}\left(\mathrm{d} s \mid\left\{\omega_{\alpha}\right\}_{i-1}\right)}{1-F_{i}^{\alpha}\left(s_{-} \mid\left\{\omega_{\alpha}\right\}_{i-1}\right)}<\infty \\
\left\{\omega_{\alpha}\right\}_{i-1} \in \Omega^{i-1}, \alpha \in X^{0} . \\
\int_{\left[a_{i}^{\alpha}, a_{i+1}^{\alpha}\right)}\left|\varphi_{i}^{\alpha}\left(s \mid\left\{\omega_{\alpha}\right\}_{i-1}\right)\right| \frac{F_{i}^{\alpha, 1}\left(\mathrm{~d} s \mid\left\{\omega_{\alpha}\right\}_{i-1}\right)}{1-F_{i}^{\alpha, 1}\left(s_{-} \mid\left\{\omega_{\alpha}\right\}_{i-1}\right)} \leqslant \\
\int_{\left[t_{i}^{\alpha}, a_{i+1}^{\alpha}\right)} \leqslant 2 C_{\alpha} \frac{F_{i}^{\alpha, 1}\left(\mathrm{~d} s \mid\left\{\omega_{\alpha}\right\}_{i-1}\right)}{1-F_{i}^{\alpha, 1}\left(s_{-} \mid\left\{\omega_{\alpha}\right\}_{i-1}\right)} \\
{\left[1-F_{i}^{\alpha}\left(s \mid\left\{\omega_{i}^{\alpha}\right]\right.\right.}
\end{gathered}
$$

because the first integral is finite and the second integral is finite since

$$
\begin{aligned}
\gamma^{i, \alpha}\left(\left\{\omega_{\alpha}\right\}_{i-1}, t\right) & =\int_{\left[a_{i}^{\alpha}, t\right]} \frac{\psi_{i}^{\alpha}\left(\left\{\omega_{\alpha}\right\}_{i-1}, \mathrm{~d} \tau\right)}{\psi_{i}^{\alpha}\left(\left\{\omega_{\alpha}\right\}_{i-1}, \tau\right)-f_{\alpha}\left(g_{i}^{\alpha}\left(\left\{\omega_{\alpha}\right\}_{i-1}, \tau\right)\right)} \\
& =\int_{\left[a_{i}^{\alpha}, t\right]} \frac{F_{i}^{\alpha, 1}\left(\mathrm{~d} \tau \mid\left\{\omega_{\alpha}\right\}_{i-1}\right)}{1-F_{i}^{\alpha, 1}\left(\tau_{-} \mid\left\{\omega_{\alpha}\right\}_{i-1}\right)} \\
= & \int_{\left[a_{i}^{\alpha}, t\right]} \frac{f_{\alpha}^{\prime}\left(T_{i}^{\alpha}\left(\tau \mid\left\{\omega_{\alpha}\right\}_{i-1}\right)\right) F_{i}^{\alpha}\left(\mathrm{d} \tau \mid\left\{\omega_{\alpha}\right\}_{i-1}\right)}{U\left(\left\{\omega_{\alpha}\right\}_{i-1}, \tau\right)\left[1-F_{i}^{\alpha}\left(\tau_{-} \mid\left\{\omega_{\alpha}\right\}_{i-1}\right)\right]}
\end{aligned}
$$


Therefore

$$
\int_{\left[t_{i}^{\alpha}, a_{i+1}^{\alpha}\right)}\left(1-F_{i}^{\alpha}\left(s \mid\left\{\omega_{\alpha}\right\}_{i-1}\right)\right)^{\varepsilon_{\alpha}^{i}} \frac{F_{i}^{\alpha, 1}\left(\mathrm{~d} s \mid\left\{\omega_{\alpha}\right\}_{i-1}\right)}{1-F_{i}^{\alpha, 1}\left(s_{-} \mid\left\{\omega_{\alpha}\right\}_{i-1}\right)} \leqslant \frac{f_{1}^{\alpha}}{\varepsilon_{\alpha}^{i} f_{2}^{\alpha}} .
$$

The theorem 5 is proved. Then we assume that interval $[a, b)$ coincides with the interval $[0, T)$, that is $a=0, b=T$. The time $T$ is the terminal time of monotonous of the option.

Definition 5. A stock market is effective on the time interval $[0, T)$, if there is a certain probability space $\{\Omega, \mathcal{F}, P\}$, constructed above, a random process $\xi_{t}^{0}\left(\left\{\alpha, \omega_{\alpha}\right\}\right)$ on $i t$, describing the evolution of the average price of stocks such that $\xi_{t}^{0}\left(\left\{\alpha, \omega_{\alpha}\right\}\right) \mathrm{e}^{-r t}$ is a non-negative uniformly integrable and non-singular martingale on $\{\Omega, \mathcal{F}, P\}$ with respect to the flow of the $\sigma$-algebras $\mathcal{F}_{t}$, where the $\sigma$-algebras $\mathcal{F}$ and $\mathcal{F}_{t}$ are the completion of the $\sigma$-algebras $\mathcal{F}^{0}$ and $\mathcal{F}_{t}^{0}$ with respect to the measure $P$ on $\mathcal{F}^{0}$, generated by the family of distribution functions $F_{i}^{\alpha}\left(\omega_{i}^{\alpha} \mid\left\{\omega_{\alpha}\right\}_{i-1}\right)$. The random process $\xi_{t}^{0}\left(\left\{\alpha, \omega_{\alpha}\right\}\right)$ has the form

$$
\begin{gathered}
\xi_{t}^{0}\left(\left\{\alpha, \omega_{\alpha}\right\}\right)=B_{0} \mathrm{e}^{r t} \sum_{i=1}^{k(\alpha)} \chi_{\left[a_{i}^{\alpha}, a_{i+1}^{\alpha}\right)}(t) \xi_{t}^{i, \alpha}\left(\left\{\omega_{\alpha}\right\}_{i}\right), \\
+\frac{\left.\xi_{t}^{i, \alpha}\left(\left\{\omega_{\alpha}\right\}_{i}\right\}\right)=g_{i}^{\alpha}\left(\left\{\omega_{\alpha}\right\}_{i}\right) \chi_{\left[a_{i}^{\alpha}, t\right]}\left(\omega_{i}^{\alpha}\right)}{1-F_{i}^{\alpha}\left(t \mid\left\{\omega_{\alpha}\right\}_{i-1}\right)} \int_{\left(t, a_{i+1}^{\alpha}\right)} g_{i}^{\alpha}\left(\left\{\omega_{\alpha}\right\}_{i}\right) F_{i}^{\alpha}\left(\mathrm{d} \omega_{i}^{\alpha} \mid\left\{\omega_{\alpha}\right\}_{i-1}\right) \chi_{\left(t, a_{i+1}^{\alpha}\right)}\left(\omega_{i}^{\alpha}\right),
\end{gathered}
$$

where $r$ is an interest rate, the evolution of price of a stock being described by a certain random process

$$
\begin{aligned}
S_{t}\left(\left\{\alpha, \omega_{\alpha}\right\}\right) & =\frac{S_{0} \mathrm{e}^{r t}}{V_{\alpha}} \sum_{i=1}^{k(\alpha)} \chi_{\left[a_{i}^{\alpha}, a_{i+1}^{\alpha}\right)}(t) f_{\alpha}\left(\xi_{t}^{i, \alpha}\left(\left\{\omega_{\alpha}\right\}_{i}\right)\right), \\
V_{\alpha} & =f_{\alpha}\left(M_{\alpha} \phi^{\alpha}\left(\left\{\omega_{\alpha}\right\}_{k(\alpha)}\right)\right)
\end{aligned}
$$

for a certain family of functions $f_{\alpha}(x)>0, x \in R^{1}, \alpha \in X^{0}$, which are strictly fulfillment, $\sup _{x \in R^{1}}\left|f_{\alpha}^{\prime}(x)\right|=f_{1}^{\alpha}<\infty, \inf _{x \in R^{1}}\left|f_{\alpha}^{\prime}(x)\right|=f_{2}^{\alpha}>0$, moreover,

$$
\sup _{i} \sup _{\left\{\omega_{\alpha}\right\}_{i-1} \in \Omega^{i-1}} \sup _{s \in\left[a_{i}^{\alpha}, a_{i+1}^{\alpha}\right)} \frac{\Delta F_{i}^{\alpha}\left(s \mid\left\{\omega_{\alpha}\right\}_{i-1}\right)}{1-F_{i}^{\alpha}\left(s_{-} \mid\left\{\omega_{\alpha}\right\}_{i-1}\right)}<\frac{f_{2}^{\alpha}}{f_{1}^{\alpha}}
$$

The limit

$$
\left.\phi^{\alpha}\left(\left\{\omega_{\alpha}\right\}_{k(\alpha)}\right)\right)=\lim _{t \rightarrow T} \xi_{t}^{0}\left(\left\{\alpha, \omega_{\alpha}\right\}\right) B_{0}^{-1} \mathrm{e}^{-r t}
$$

satisfies the conditions:

1) $\left|\phi^{\alpha}\left(\left\{\omega_{\alpha}\right\}_{k(\alpha)}\right)\right| \leqslant C_{\alpha}<\infty, \quad \alpha \in X^{0}, \int_{X^{0}} C_{\alpha} \mathrm{d} \mu(\alpha)<\infty$;

2) there exists $t_{i}^{\alpha} \in\left[a_{i}^{\alpha}, a_{i+1}^{\alpha}\right)$ such that

$$
\left|\phi^{\alpha}\left(\left\{\omega_{\alpha}\right\}_{i-1}, s_{1},\left\{\omega_{\alpha}\right\}_{[i+1, k(\alpha)]}\right)-\phi^{\alpha}\left(\left\{\omega_{\alpha}\right\}_{i-1}, s_{2},\left\{\omega_{\alpha}\right\}_{[i+1, k(\alpha)]}\right)\right| \leqslant
$$




$$
\begin{gathered}
\leqslant C_{i}^{\alpha}\left|F_{i}^{\alpha}\left(s_{1} \mid\left\{\omega_{\alpha}\right\}_{i-1}\right)-F_{i}^{\alpha}\left(s_{2} \mid\left\{\omega_{\alpha}\right\}_{i-1}\right)\right|^{\varepsilon_{\alpha}^{i}}, \varepsilon_{\alpha}^{i}>0, s_{1}, s_{2} \in\left[t_{i}^{\alpha}, a_{i+1}^{\alpha}\right), \\
\left.C_{i}^{\alpha}<\infty, i=\overline{1, k(\alpha)}, \quad\left\{\omega_{\alpha}\right\}_{i-1} \in \Omega^{i-1},\left\{\omega_{\alpha}\right\}_{[i+1, k(\alpha)]}\right) \in \Omega^{k(\alpha)-i}, \alpha \in X^{0} .
\end{gathered}
$$

Let us consider an economic agent on the stock market, who acts an as investor, that is, he or she wants to multiply his or her capital using the possibilities of the stock market. We assume that the stock market is effective and the evolution of a stock price occurs according to the formula (25). We assume that the evolution of non-risky active price occurs according to the law

$$
B(t)=B_{0} \mathrm{e}^{r t}
$$

where $r$ is an interest rate, $B_{0}$ is an initial capital of the investor on a deposit.

Definition 6. A stochastic process $\delta_{t}\left(\left\{\alpha, \omega_{\alpha}\right\}\right)$ belongs to the class $A_{0}$, if

$$
\begin{gathered}
\delta_{t}\left(\left\{\alpha, \omega_{\alpha}\right\}\right)=\sum_{i=1}^{k(\alpha)} \chi_{\left[a_{i}^{\alpha}, a_{i+1}^{\alpha}\right)}(t) \delta_{t}^{i, \alpha}\left(\left\{\omega_{\alpha}\right\}_{i}\right), \\
\delta_{t}^{i, \alpha}\left(\left\{\omega_{\alpha}\right\}_{i}\right)=b_{i}^{\alpha, 1}\left(\left\{\omega_{\alpha}\right\}_{i}, t\right) \chi_{\left[a_{i}^{\alpha}, t\right]}\left(\omega_{i}^{\alpha}\right)+b_{i}^{\alpha, 2}\left(\left\{\omega_{\alpha}\right\}_{i-1}, t\right) \chi_{\left(t, a_{i+1}^{\alpha}\right)}\left(\omega_{i}^{\alpha}\right),
\end{gathered}
$$

$b_{i}^{\alpha, 1}\left(\left\{\omega_{\alpha}\right\}_{i}, t\right)$ is a measurable mapping from the measurable space $\left\{\Omega^{i}, \overline{\mathcal{F}}_{i}^{0}\right\}$ to the measurable space $\left\{R^{1}, \mathcal{B}\left(R^{1}\right)\right\}$ at every fixed $t$ from the interval $[0, T), b_{i}^{\alpha, 2}\left(\left\{\omega_{\alpha}\right\}_{i-1}, t\right)$ is a measurable mapping from the measurable space $\left\{\Omega^{i-1}, \overline{\mathcal{F}}_{i-1}^{0}\right\}$ to the measurable space $\left\{R^{1}, \mathcal{B}\left(R^{1}\right)\right\}$ at every fixed $t \in[0, T)$. Moreover, $b_{i}^{\alpha, 1}\left(\left\{\omega_{\alpha}\right\}_{i}, t\right)$ is a bounded measurable mapping from the measurable space $\{[0, T), \mathcal{B}([0, T))\}$ to the measurable space $\left\{R^{1}, \mathcal{B}\left(R^{1}\right)\right\}$ at every fixed $\left\{\omega_{\alpha}\right\}_{i} \in \Omega^{i}, b_{i}^{\alpha, 2}\left(\left\{\omega_{\alpha}\right\}_{i-1}, t\right)$ is a bounded measurable mapping from $\{[0, T), \mathcal{B}([0, T))\}$ to $\left\{R^{1}, \mathcal{B}\left(R^{1}\right)\right\}$ at every fixed $\left\{\omega_{\alpha}\right\}_{i-1} \in \Omega^{i-1}$.

Let the capital of an investor $X_{t}\left(\left\{\alpha, \omega_{\alpha}\right\}\right)$ at time $t$ equal

$$
X_{t}\left(\left\{\alpha, \omega_{\alpha}\right\}\right)=B(t) \beta_{t}\left(\left\{\alpha, \omega_{\alpha}\right\}\right)+\gamma_{t}\left(\left\{\alpha, \omega_{\alpha}\right\}\right) S_{t}\left(\left\{\alpha, \omega_{\alpha}\right\}\right),
$$

where the stochastic processes $\beta_{t}\left(\left\{\alpha, \omega_{\alpha}\right\}\right)$ and $\gamma_{t}\left(\left\{\alpha, \omega_{\alpha}\right\}\right)$ belong to the class $A_{0}$. The pair $\pi_{t}=\left\{\beta_{t}\left(\left\{\alpha, \omega_{\alpha}\right\}\right), \gamma_{t}\left(\left\{\alpha, \omega_{\alpha}\right\}\right)\right\}$ is called the financial strategy of the investor. The capital of the investor with the financial strategy $\pi_{t}$ will be denoted by $X_{t}^{\pi}\left(\left\{\alpha, \omega_{\alpha}\right\}\right)$.

Definition 7. A financial strategy $\pi_{t}=\left\{\beta_{t}\left(\left\{\alpha, \omega_{\alpha}\right\}\right), \gamma_{t}\left(\left\{\alpha, \omega_{\alpha}\right\}\right)\right\}$ of an investor is called self-financing if the random processes $\beta_{t}\left(\left\{\alpha, \omega_{\alpha}\right\}\right)$ and $\gamma_{t}\left(\left\{\alpha, \omega_{\alpha}\right\}\right)$ belong to the class $A_{0}$, for the investor capital $X_{t}^{\pi}\left(\left\{\alpha, \omega_{\alpha}\right\}\right)$ the representation

$$
X_{t}^{\pi}\left(\left\{\alpha, \omega_{\alpha}\right\}\right)=X_{0}^{\pi}(\alpha)+\int_{[0, t]} \beta_{\tau}\left(\left\{\alpha, \omega_{\alpha}\right\}\right) \mathrm{d} B(\tau)+\int_{[0, t]} \gamma_{\tau}\left(\left\{\alpha, \omega_{\alpha}\right\}\right) \mathrm{d} S_{\tau}\left(\left\{\alpha, \omega_{\alpha}\right\}\right)
$$

is valid, the discounted capital

$$
Y_{t}^{\pi}\left(\left\{\alpha, \omega_{\alpha}\right\}\right)=\frac{X_{t}^{\pi}\left(\left\{\alpha, \omega_{\alpha}\right\}\right)}{B(t)}
$$


belongs to the class of local martingale on the probability space $\left\{\Omega, \mathcal{F}_{1}, P_{1}\right\}$ with respect to the flow of the $\sigma$-algebras $\mathcal{F}_{t}^{1}, M^{1}\left|X_{t}^{\pi}\left(\left\{\alpha, \omega_{\alpha}\right\}\right)\right|<\infty$, where $\mathcal{F}_{1}, P_{1}$ and $\mathcal{F}_{t}^{1}$ are constructed in the theorem 5.

A class of self-financing strategy is denoted by $S F$.

Lemma 10. Let a financial strategy $\pi_{t}=\left\{\beta_{t}\left(\left\{\alpha, \omega_{\alpha}\right\}\right), \gamma_{t}\left(\left\{\alpha, \omega_{\alpha}\right\}\right)\right\}$ be self-financing, then for the investor capital the representations

$$
\begin{gathered}
X_{t}^{\pi}\left(\left\{\alpha, \omega_{\alpha}\right\}\right)=X_{0}^{\pi}(\alpha)+\int_{[0, t]} \beta_{\tau}\left(\left\{\alpha, \omega_{\alpha}\right\}\right) \mathrm{d} B(\tau)+\int_{[0, t]} \gamma_{\tau}\left(\left\{\alpha, \omega_{\alpha}\right\}\right) \mathrm{d} S_{\tau}\left(\left\{\alpha, \omega_{\alpha}\right\}\right) \\
X_{t}^{\pi}\left(\left\{\alpha, \omega_{\alpha}\right\}\right)=\mathrm{e}^{r t} X_{0}^{\pi}(\alpha)+B_{0} \mathrm{e}^{r t} \int_{[0, t]} \gamma_{\tau}\left(\left\{\alpha, \omega_{\alpha}\right\}\right) \mathrm{d} S_{\tau}^{0}\left(\left\{\alpha, \omega_{\alpha}\right\}\right)
\end{gathered}
$$

are equivalent, where

$$
S_{t}^{0}\left(\left\{\alpha, \omega_{\alpha}\right\}\right)=\frac{S_{0}}{B_{0} V_{\alpha}} \sum_{i=1}^{k(\alpha)} \chi_{\left[a_{i}^{\alpha}, a_{i+1}^{\alpha}\right)}(t) f_{\alpha}\left(\xi_{t}^{i, \alpha}\left(\left\{\omega_{\alpha}\right\}_{i}\right)\right) .
$$

Proof. Since $X_{t}^{\pi}\left(\left\{\alpha, \omega_{\alpha}\right\}\right)$ is a process of a bounded variation on any interval $[0, t]$, therefore from (31) and lemma 1

$$
\begin{gathered}
X_{t}^{\pi}\left(\left\{\alpha, \omega_{\alpha}\right\}\right)=X_{0}^{\pi}(\alpha)+\int_{[0, t]}\left[X_{0}^{\alpha}+B_{0} \int_{[0, t]} \gamma_{\tau}\left(\left\{\alpha, \omega_{\alpha}\right\}\right) \mathrm{d} S_{\tau}^{0}\left(\left\{\alpha, \omega_{\alpha}\right\}\right)\right] \mathrm{de}^{r t} \\
+B_{0} \int_{[0, t]} \mathrm{e}^{r \tau} \gamma_{\tau}\left(\left\{\alpha, \omega_{\alpha}\right\}\right) \mathrm{d} S_{\tau}^{0}\left(\left\{\alpha, \omega_{\alpha}\right\}\right) \\
=X_{0}^{\pi}(\alpha)+\int_{[0, t]} X_{\tau}\left(\left\{\alpha, \omega_{\alpha}\right\}\right) \frac{\mathrm{d} B(\tau)}{B(\tau)}+\int_{[0, t]} B(\tau) \gamma_{\tau}\left(\left\{\alpha, \omega_{\alpha}\right\}\right) \mathrm{d} S_{\tau}^{0}\left(\left\{\alpha, \omega_{\alpha}\right\}\right) .
\end{gathered}
$$

Since

$$
\begin{gathered}
S_{t}\left(\left\{\alpha, \omega_{\alpha}\right\}\right)=B(t) S_{t}^{0}\left(\left\{\alpha, \omega_{\alpha}\right\}\right), \\
\mathrm{d} S_{t}\left(\left\{\alpha, \omega_{\alpha}\right\}\right)=S_{t}^{0}\left(\left\{\alpha, \omega_{\alpha}\right\}\right) \mathrm{d} B(t)+B(t) \mathrm{d} S_{t}^{0}\left(\left\{\alpha, \omega_{\alpha}\right\}\right),
\end{gathered}
$$

therefore, taking into account (28) and (33), we obtain

$$
\begin{gathered}
X_{t}^{\pi}\left(\left\{\alpha, \omega_{\alpha}\right\}\right)=X_{0}^{\pi}(\alpha)+\int_{[0, t]} \beta_{\tau}\left(\left\{\alpha, \omega_{\alpha}\right\}\right) \mathrm{d} B(\tau)+\int_{[0, t]} \frac{\mathrm{d} B(\tau)}{B(\tau)} \gamma_{\tau}\left(\left\{\alpha, \omega_{\alpha}\right\}\right) S_{\tau}\left(\left\{\alpha, \omega_{\alpha}\right\}\right) \\
+\int_{[0, t]} \gamma_{\tau}\left(\left\{\alpha, \omega_{\alpha}\right\}\right) \mathrm{d} S_{\tau}\left(\left\{\alpha, \omega_{\alpha}\right\}\right)-\int_{[0, t]} \gamma_{\tau}\left(\left\{\alpha, \omega_{\alpha}\right\}\right) S_{\tau}^{0}\left(\left\{\alpha, \omega_{\alpha}\right\}\right) \mathrm{d} B(\tau)
\end{gathered}
$$




$$
=X_{0}^{\pi}(\alpha)+\int_{[0, t]} \beta_{\tau}\left(\left\{\alpha, \omega_{\alpha}\right\}\right) \mathrm{d} B(\tau)+\int_{[0, t]} \gamma_{\tau}\left(\left\{\alpha, \omega_{\alpha}\right\}\right) \mathrm{d} S_{\tau}\left(\left\{\alpha, \omega_{\alpha}\right\}\right) .
$$

This proves the lemma 10 in one direction. Applying the same argument in the inverse direction we obtain the proof of the lemma 10.

Denote by $S F^{R}$ a set of self-financing strategies satisfying the conditions

$$
M^{1}\left\{Y_{t}^{\pi}\left(\left\{\alpha, \omega_{\alpha}\right\}\right) \mid \mathcal{F}_{t}^{1}\right\} \geqslant-M^{1}\left\{R \mid \mathcal{F}_{t}^{1}\right\}, \quad M^{1} R<\infty,
$$

where $R$ is a non-negative random value on $\left\{\Omega, \mathcal{F}_{1}, P_{1}\right\}$.

Lemma 11. Let $\pi_{t}=\left\{\beta_{t}\left(\left\{\alpha, \omega_{\alpha}\right\}\right), \gamma_{t}\left(\left\{\alpha, \omega_{\alpha}\right\}\right)\right\}$ be a self-financing strategy, that is, $\pi_{t} \in S F^{R}$, then $\left\{Y_{t}^{\pi}, \mathcal{F}_{t}^{1}, t \in[0, T]\right\}$ is a supermartingale and for any stop time $\tau_{1}$ and $\tau_{2}$ such that $P_{1}\left(\tau_{1} \leqslant \tau_{2}\right)=1$ the inequality

$$
M^{1}\left\{Y_{\tau_{2}}^{\pi}\left(\left\{\alpha, \omega_{\alpha}\right\}\right) \mid \mathcal{F}_{\tau_{1}}^{1}\right\} \leqslant Y_{\tau_{1}}^{\pi}\left(\left\{\alpha, \omega_{\alpha}\right\}\right)
$$

is valid.

The proof is similar to the proof of the analogous lemma in [2].

Corollary 3. If $\pi_{t} \in S F^{R}$, then for any stop time $\tau \geqslant 0, P_{1}(\tau<\infty)=1$

$$
M^{1} Y_{\tau}^{\pi}\left(\left\{\alpha, \omega_{\alpha}\right\}\right) \leqslant Y_{0}^{\pi}(\alpha)=\frac{X_{0}^{\pi}(\alpha)}{B_{0}}
$$

Definition 8. A self-financing strategy $\pi_{t}$ is an arbitrage strategy on $[0, T]$, if from that

$$
X_{0}^{\pi}(\alpha) \leqslant 0, \quad X_{T}^{\pi}\left(\left\{\alpha, \omega_{\alpha}\right\}\right) \geqslant 0
$$

it follows that $X_{T}^{\pi}\left(\left\{\alpha, \omega_{\alpha}\right\}\right)>0$ with a positive probability.

Lemma 12. Any strategy $\pi_{t} \in S F^{R}$, where $R$ is non-negative and integrable random value on probability space, is not arbitrage strategy.

The proof of the lemma is analogous to the proof of the similar lemma in [2]. Let $\phi_{T}=\phi_{T}\left(\left\{\alpha, \omega_{\alpha}\right\}\right)=\phi_{T}^{\alpha}\left(\left\{\omega_{\alpha}\right\}_{k(\alpha)}\right)$ be $\mathcal{F}^{0}$ measurable random value on the probability space $\left\{\Omega, \mathcal{F}^{0}, P\right\}$.

Definition 9. A self-financing strategy $\pi_{t} \in S F^{R}$ is $\left(x^{\alpha}, \phi_{T}\right)$-hedge for the European type option if the capital $X_{t}^{\pi}\left(\left\{\alpha, \omega_{\alpha}\right\}\right)$, corresponding to this strategy is such that $X_{0}^{\pi}(\alpha)=x^{\alpha}$ and with probability 1 with respect to the measure $P_{1}$

$$
X_{T}^{\pi}\left(\left\{\alpha, \omega_{\alpha}\right\}\right) \geqslant \phi_{T}\left(\left\{\alpha, \omega_{\alpha}\right\}\right) .
$$

$\left(x^{\alpha}, \phi_{T}\right)$-hedge $\pi_{t}^{*} \in S F^{R}$ is called minimal if for any $\left(x^{\alpha}, \phi_{T}\right)$-hedge $\pi_{t} \in S F^{R}$ the inequality

$$
X_{T}^{\pi}\left(\left\{\alpha, \omega_{\alpha}\right\}\right) \geqslant X_{T}^{\pi^{*}}\left(\left\{\alpha, \omega_{\alpha}\right\}\right)
$$

is valid. 
Then we consider self-financing strategies, belonging to $S F^{0}$, that is, in this case $X_{t}^{\pi}\left(\left\{\alpha, \omega_{\alpha}\right\}\right) \geqslant 0$.

Definition 10. Let $H_{T}\left(x^{\alpha}, \phi_{T}\right)$ be the set of $\left(x^{\alpha}, \phi_{T}\right)$-hedges from $S F^{0}$. Investment value is called the value

$$
C_{T}^{\alpha}\left(\phi_{T}\right)=\inf \left\{x^{\alpha} \geqslant 0, H_{T}\left(x^{\alpha}, \phi_{T}\right) \neq \emptyset\right\}, \alpha \in X^{0},
$$

where $\emptyset$ is the empty set.

The main problem is to calculate $C_{T}^{\alpha}\left(\phi_{T}\right)$ and to find an expression for the portfolio of an investor $\pi_{t}^{*}$ at every moment of time $t$ the initial capital of which is $x^{\alpha}$.

Further on we assume that $T<\infty$, then

$$
\lim _{t \rightarrow T} S_{t}\left(\left\{\alpha, \omega_{\alpha}\right\}\right)=S_{T}\left(\left\{\alpha, \omega_{\alpha}\right\}\right)=\frac{S_{0} \mathrm{e}^{r T}}{V_{\alpha}} f_{\alpha}\left(\phi\left(\left\{\alpha, \omega_{\alpha}\right\}\right)\right) .
$$

Theorem 6. Let a stock market be effective, the evolution of a risky active price comes according to the formula (25) and the evolution of non-risky active price occur by (27). If $f(x)$ is a certain function such that $\left|f\left(x_{1}\right)-f\left(x_{2}\right)\right| \leqslant C\left|x_{1}-x_{2}\right|$ and the paying function at terminal time $T$ is given by the formula

$$
f_{T}\left(\left\{\alpha, \omega_{\alpha}\right\}\right)=f\left(S_{T}\left(\left\{\alpha, \omega_{\alpha}\right\}\right)\right),
$$

moreover, the conditions

$$
\int_{X^{0}} \frac{f_{1}^{\alpha} C_{\alpha}}{V_{\alpha}} \mathrm{d} \mu(\alpha)<\infty, \int_{X^{0}} \frac{f_{\alpha}(0)}{V_{\alpha}} \mathrm{d} \mu(\alpha)<\infty,
$$

are valid, then the minimal hedge $\pi_{t}^{*}$ exists, evolution of the capital investor $X_{t}^{*}\left(\left\{\alpha, \omega_{\alpha}\right\}\right)$, option price $X_{0}^{*}(\alpha)$ and self-financial strategy $\left\{\beta_{t}^{*}\left(\left\{\alpha, \omega_{\alpha}\right\}\right)\right.$, $\left.\gamma_{t}^{*}\left(\left\{\alpha, \omega_{\alpha}\right\}\right)\right\}$ corresponding to the minimal hedge $\pi_{t}^{*}$ are given by the formulas

$$
\begin{gathered}
X_{t}^{*}\left(\left\{\alpha, \omega_{\alpha}\right\}\right)=\mathrm{e}^{r(t-T)} M^{1}\left\{f\left(S_{T}\left(\left\{\alpha, \omega_{\alpha}\right\}\right)\right) \mid \mathcal{F}_{t}^{1}\right\}, \\
X_{0}^{*}(\alpha)=\mathrm{e}^{-r T} M_{\alpha}^{1} f\left(S_{T}\left(\left\{\alpha, \omega_{\alpha}\right\}\right)\right), \quad \gamma_{t}^{*}\left(\left\{\alpha, \omega_{\alpha}\right\}\right)=\psi_{k(\alpha)}\left(t \mid\{\omega\}_{\alpha}\right), \\
\beta_{t}^{*}\left(\left\{\alpha, \omega_{\alpha}\right\}\right)=\frac{X_{t}^{*}\left(\left\{\alpha, \omega_{\alpha}\right\}\right)-\gamma_{t}^{*}\left(\left\{\alpha, \omega_{\alpha}\right\}\right) S_{t}\left(\left\{\alpha, \omega_{\alpha}\right\}\right)}{B(t)},
\end{gathered}
$$

where

$$
\begin{aligned}
\psi_{k(\alpha)}\left(s \mid \omega_{\alpha}\right)= & \sum_{i=1}^{k(\alpha)} \chi_{\left[a_{i}^{\alpha}, a_{i+1}^{\alpha}\right)}(s) \frac{\varphi_{i}^{\alpha}\left(s \mid\left\{\omega_{\alpha}\right\}_{i-1}\right)}{\varphi_{i}^{0, \alpha}\left(s \mid\left\{\omega_{\alpha}\right\}_{i-1}\right)}, \\
& \varphi_{i}^{0, \alpha}\left(s \mid\left\{\omega_{\alpha}\right\}_{i-1}\right)= \\
= & f_{\alpha}\left(g_{i}^{\alpha}\left(\left\{\omega_{\alpha}\right\}_{i-1}, s\right)\right) \\
-\frac{1}{1-F_{i}^{\alpha, 1}\left(s \mid\left\{\omega_{\alpha}\right\}_{i-1}\right)} & \int_{\left(s, a_{i+1}^{\alpha}\right)} f_{\alpha}\left(g_{i}^{\alpha}\left(\left\{\omega_{\alpha}\right\}_{i}\right)\right) F_{i}^{\alpha, 1}\left(\mathrm{~d} \omega_{i}^{\alpha} \mid\left\{\omega_{\alpha}\right\}_{i-1}\right),
\end{aligned}
$$




$$
\begin{gathered}
\varphi_{i}^{\alpha}\left(s \mid\left\{\omega_{\alpha}\right\}_{i-1}\right)= \\
=\bar{\phi}_{i}^{\alpha}\left(\left\{\omega_{\alpha}\right\}_{i-1}, s\right)-\frac{1}{1-F_{i}^{\alpha, 1}\left(s \mid\left\{\omega_{\alpha}\right\}_{i-1}\right)} \int_{\left(s, a_{i+1}^{\alpha}\right)} \bar{\phi}_{i}^{\alpha}\left(\left\{\omega_{\alpha}\right\}_{i}\right) F_{i}^{\alpha, 1}\left(\mathrm{~d} \omega_{i}^{\alpha} \mid\left\{\omega_{\alpha}\right\}_{i-1}\right), \\
\bar{\phi}_{i}^{\alpha}\left(\left\{\omega_{\alpha}\right\}_{i}\right)=\frac{1}{B_{0} \mathrm{r}^{r T}} \int_{\Omega_{i+1}} \ldots \int_{\Omega_{k(\alpha)}} f\left(S_{0} \mathrm{e}^{r T} V_{\alpha}^{-1} f_{\alpha}\left(\phi^{\alpha}\left(\left\{\omega_{\alpha}\right\}_{i},\left\{\omega_{\alpha}\right\}_{[i+1, k(\alpha)]}\right)\right)\right) \\
\times F_{i+1}^{\alpha, 1}\left(\mathrm{~d} \omega_{i+1}^{\alpha} \mid\left\{\omega_{\alpha}\right\}_{i}\right) \times \ldots \times F_{k(\alpha)}^{\alpha, 1}\left(\mathrm{~d} \omega_{k(\alpha)}^{\alpha} \mid\left\{\omega_{\alpha}\right\}_{k(\alpha)-1}\right) .
\end{gathered}
$$

Proof. To prove the theorem 6 it is sufficient to verify the monotonous of the conditions of the theorem 5. Since

$$
S_{T}\left(\left\{\alpha, \omega_{\alpha}\right\}\right)=\frac{S_{0} \mathrm{e}^{r T}}{V_{\alpha}} f_{\alpha}\left(\phi\left(\left\{\alpha, \omega_{\alpha}\right\}\right)\right),
$$

then

$$
\begin{gathered}
\frac{f\left(S_{T}\left(\left\{\alpha, \omega_{\alpha}\right\}\right)\right)}{B_{0} \mathrm{e}^{\mathrm{T}}} \leqslant \\
\leqslant \frac{1}{B_{0} \mathrm{e}^{r T}}\left[f(0)+C S_{0} \mathrm{e}^{r T} \frac{f_{\alpha}(0)}{V_{\alpha}}+C S_{0} \mathrm{e}^{r T} \frac{f_{1}^{\alpha} C_{\alpha}}{V_{\alpha}}\right]=C_{\alpha}^{\prime}, \quad \int_{X^{0}} C_{\alpha}^{\prime} d \mu(\alpha)<\infty . \\
\left|f\left(f_{\alpha}\left(\phi^{\alpha}\left(\left\{\omega_{\alpha}\right\}_{i-1}, s_{1},\left\{\omega_{\alpha}\right\}_{[i+1, k(\alpha)]}\right)\right)\right)-f\left(f_{\alpha}\left(\phi^{\alpha}\left(\left\{\omega_{\alpha}\right\}_{i-1}, s_{2},\left\{\omega_{\alpha}\right\}_{[i+1, k(\alpha)]}\right)\right)\right)\right| \leqslant \\
\leqslant C f_{1}^{\alpha} C_{i}^{\alpha}\left|F_{i}^{\alpha}\left(s_{1} \mid\left\{\omega_{\alpha}\right\}_{i-1}\right)-F_{i}^{\alpha}\left(s_{2} \mid\left\{\omega_{\alpha}\right\}_{i-1}\right)\right|^{\varepsilon_{\alpha}^{i}}, \varepsilon_{\alpha}^{i}>0, s_{1}, s_{2} \in\left[t_{i}^{\alpha}, a_{i+1}^{\alpha}\right), \\
\left.i=\overline{1, k(\alpha)},\left\{\omega_{\alpha}\right\}_{i-1} \in \Omega^{i-1},\left\{\omega_{\alpha}\right\}_{[i+1, k(\alpha)]}\right) \in \Omega^{k(\alpha)-i}, \alpha \in X^{0} .
\end{gathered}
$$

Further, $\xi_{t}^{0}\left(\left\{\alpha, \omega_{\alpha}\right\}\right) \mathrm{e}^{r T}$ is a non-negative martingale on $\{\Omega, \mathcal{F}, P\}$ satisfying conditions:

$$
\sup _{\left\{\omega_{\alpha}\right\}_{i} \in \Omega^{i}}\left|g_{i}^{\alpha}\left(\left\{\omega_{\alpha}\right\}_{i}\right)\right|=\beta_{i}^{\alpha} \leqslant C_{\alpha}<\infty, i=\overline{1, k(\alpha)}, \alpha \in X^{0},
$$

moreover, since

$$
\begin{gathered}
g_{i}^{\alpha}\left(\left\{\omega_{\alpha}\right\}_{i}\right)=\int_{\Omega_{i+1}} \ldots \int_{\Omega_{k(\alpha)}} \phi^{\alpha}\left(\left\{\omega_{\alpha}\right\}_{i},\left\{\omega_{\alpha}\right\}_{[i+1, k(\alpha)]}\right) \\
\times F_{i+1}^{\alpha}\left(\mathrm{d} \omega_{i+1}^{\alpha} \mid\left\{\omega_{\alpha}\right\}_{i}\right) \times \ldots \times F_{k(\alpha)}^{\alpha}\left(\mathrm{d} \omega_{k(\alpha)}^{\alpha} \mid\left\{\omega_{\alpha}\right\}_{k(\alpha)-1}\right) . \\
\left|\phi^{\alpha}\left(\left\{\omega_{\alpha}\right\}_{i-1}, s_{1},\left\{\omega_{\alpha}\right\}_{[i+1, k(\alpha)]}\right)-\phi^{\alpha}\left(\left\{\omega_{\alpha}\right\}_{i-1}, s_{2},\left\{\omega_{\alpha}\right\}_{[i+1, k(\alpha)]}\right)\right| \leqslant \\
\leqslant C_{i}^{\alpha}\left|F_{i}^{\alpha}\left(s_{1} \mid\left\{\omega_{\alpha}\right\}_{i-1}\right)-F_{i}^{\alpha}\left(s_{2} \mid\left\{\omega_{\alpha}\right\}_{i-1}\right)\right|^{\varepsilon_{\alpha}^{i}}, \varepsilon_{\alpha}^{i}>0, s_{1}, s_{2} \in\left[t_{i}^{\alpha}, a_{i+1}^{\alpha}\right), \\
C_{i}^{\alpha}<\infty, i=\overline{1, k(\alpha)},\left\{\omega_{\alpha}\right\}_{i-1} \in \Omega^{i-1},\left\{\omega_{\alpha}\right\}_{[i+1, k(\alpha)]} \in \Omega^{k(\alpha)-i}, \alpha \in X^{0},
\end{gathered}
$$


therefore

$$
\begin{aligned}
& \int_{\left[a_{i}^{\alpha}, a_{i+1}^{\alpha}\right)}\left|\rho_{i}^{0, \alpha}\left(s \mid\left\{\omega_{\alpha}\right\}_{i-1}\right)\right| \frac{F_{i}^{\alpha}\left(\mathrm{d} s \mid\left\{\omega_{\alpha}\right\}_{i-1}\right)}{1-F_{i}^{\alpha}\left(s_{-} \mid\left\{\omega_{\alpha}\right\}_{i-1}\right)}<\infty,\left\{\omega_{\alpha}\right\}_{i-1} \in \Omega^{i-1}, \alpha \in X^{0}, \\
& \rho_{i}^{0, \alpha}\left(s \mid\left\{\omega_{\alpha}\right\}_{i-1}\right)= \\
& =g_{i}^{\alpha}\left(\left\{\omega_{\alpha}\right\}_{i-1}, s\right)-\frac{1}{1-F_{i}^{\alpha}\left(s \mid\left\{\omega_{\alpha}\right\}_{i-1}\right)} \int_{\left(s, a_{i+1}^{\alpha}\right)} g_{i}^{\alpha}\left(\left\{\omega_{\alpha}\right\}_{i}\right) F_{i}^{\alpha}\left(\mathrm{d} \omega_{i}^{\alpha} \mid\left\{\omega_{\alpha}\right\}_{i-1}\right) .
\end{aligned}
$$

Hence it follows that for the regular martingale

$$
M^{1}\left\{\frac{f\left(S_{T}\left(\left\{\alpha, \omega_{\alpha}\right\}\right)\right)}{B_{0} \mathrm{e}^{r T}} \mid \mathcal{F}_{t}^{1}\right\}
$$

the representation

$$
\begin{gathered}
M^{1}\left\{\frac{f\left(S_{T}\left(\left\{\alpha, \omega_{\alpha}\right\}\right)\right)}{B_{0} \mathrm{e}^{r T}} \mid \mathcal{F}_{t}^{1}\right\}= \\
=M_{\alpha}^{1} \frac{f\left(S_{T}\left(\left\{\alpha, \omega_{\alpha}\right\}\right)\right)}{B_{0} \mathrm{e}^{r T}}+\int_{[a, t]} \psi_{k(\alpha)}\left(\tau \mid \omega_{\alpha}\right) \mathrm{d} \bar{S}_{\tau}^{0}\left(\left\{\alpha, \omega_{\alpha}\right\}\right), t \in[a, b)
\end{gathered}
$$

is valid, where

$$
\begin{aligned}
& \psi_{k(\alpha)}\left(s \mid \omega_{\alpha}\right)=\sum_{i=1}^{k(\alpha)} \chi_{\left[a_{i}^{\alpha}, a_{i+1}^{\alpha}\right)}(s) \frac{\varphi_{i}^{\alpha}\left(s \mid\left\{\omega_{\alpha}\right\}_{i-1}\right)}{\varphi_{i}^{0, \alpha}\left(s \mid\left\{\omega_{\alpha}\right\}_{i-1}\right)}, \\
& \varphi_{i}^{0, \alpha}\left(s \mid\left\{\omega_{\alpha}\right\}_{i-1}\right)= \\
& =f_{\alpha}\left(g_{i}^{\alpha}\left(\left\{\omega_{\alpha}\right\}_{i-1}, s\right)\right) \\
& -\frac{1}{1-F_{i}^{\alpha, 1}\left(s \mid\left\{\omega_{\alpha}\right\}_{i-1}\right)} \int_{\left(s, a_{i+1}^{\alpha}\right)} f_{\alpha}\left(g_{i}^{\alpha}\left(\left\{\omega_{\alpha}\right\}_{i}\right)\right) F_{i}^{\alpha, 1}\left(\mathrm{~d} \omega_{i}^{\alpha} \mid\left\{\omega_{\alpha}\right\}_{i-1}\right), \\
& \varphi_{i}^{\alpha}\left(s \mid\left\{\omega_{\alpha}\right\}_{i-1}\right)= \\
& =\bar{\phi}_{i}^{\alpha}\left(\left\{\omega_{\alpha}\right\}_{i-1}, s\right)-\frac{1}{1-F_{i}^{\alpha, 1}\left(s \mid\left\{\omega_{\alpha}\right\}_{i-1}\right)} \int_{\left(s, a_{i+1}^{\alpha}\right)} \bar{\phi}_{i}^{\alpha}\left(\left\{\omega_{\alpha}\right\}_{i}\right) F_{i}^{\alpha, 1}\left(\mathrm{~d} \omega_{i}^{\alpha} \mid\left\{\omega_{\alpha}\right\}_{i-1}\right), \\
& \bar{\phi}_{i}^{\alpha}\left(\left\{\omega_{\alpha}\right\}_{i}\right)=\frac{1}{B_{0} \mathrm{e}^{r T}} \int_{\Omega_{i+1}} \ldots \int_{\Omega_{k(\alpha)}} f\left(S_{0} \mathrm{e}^{r T} V_{\alpha}^{-1} f_{\alpha}\left(\phi^{\alpha}\left(\left\{\omega_{\alpha}\right\}_{i},\left\{\omega_{\alpha}\right\}_{[i+1, k(\alpha)]}\right)\right)\right) \\
& \times F_{i+1}^{\alpha, 1}\left(\mathrm{~d} \omega_{i+1}^{\alpha} \mid\left\{\omega_{\alpha}\right\}_{i}\right) \times \ldots \times F_{k(\alpha)}^{\alpha, 1}\left(\mathrm{~d} \omega_{k(\alpha)}^{\alpha} \mid\left\{\omega_{\alpha}\right\}_{k(\alpha)-1}\right) .
\end{aligned}
$$

$\bar{S}_{t}^{0}\left(\left\{\alpha, \omega_{\alpha}\right\}\right)$ is a modification of

$$
S_{t}^{0}\left(\left\{\alpha, \omega_{\alpha}\right\}\right)=\frac{S_{0}}{B_{0} V_{\alpha}} \sum_{i=1}^{k(\alpha)} \chi_{\left[a_{i}^{\alpha}, a_{i+1}^{\alpha}\right)}(t) f_{\alpha}\left(\xi_{t}^{i, \alpha}\left(\left\{\omega_{\alpha}\right\}_{i}\right)\right) .
$$


such that $\bar{S}_{t}^{0}\left(\left\{\alpha, \omega_{\alpha}\right\}\right)$ is a regular martingale on the probability space $\left\{\Omega, \mathcal{F}_{1}, P_{1}\right\}$, where $\mathcal{F}_{1}$ is the completion of $\mathcal{F}^{0}$ with respect to the measure $P_{1}$, generated by the family of distributions $F_{i}^{\alpha, 1}\left(\omega_{i}^{\alpha} \mid\left\{\omega_{\alpha}\right\}_{i-1}\right), i=\overline{1, k(\alpha)}, \quad \alpha \in X^{0}$ and

$$
\begin{gathered}
\left.f_{\alpha}\left(\xi_{t}^{i, \alpha}\left(\left\{\omega_{\alpha}\right\}_{i}\right\}\right)\right)=f_{\alpha}\left(g_{i}^{\alpha}\left(\left\{\omega_{\alpha}\right\}_{i}\right)\right) \chi_{\left[a_{i}^{\alpha}, t\right]}\left(\omega_{i}^{\alpha}\right) \\
+\frac{1}{1-F_{i}^{\alpha, 1}\left(t \mid\left\{\omega_{\alpha}\right\}_{i-1}\right)} \int_{\left(t, a_{i+1}^{\alpha}\right)} f_{\alpha}\left(g_{i}^{\alpha}\left(\left\{\omega_{\alpha}\right\}_{i}\right)\right) F_{i}^{\alpha, 1}\left(\mathrm{~d} \omega_{i}^{\alpha} \mid\left\{\omega_{\alpha}\right\}_{i-1}\right) \chi_{\left(t, a_{i+1}^{\alpha}\right)}\left(\omega_{i}^{\alpha}\right), \\
i=\overline{1, k(\alpha)}, \quad \alpha \in X^{0} .
\end{gathered}
$$

The latter means that for the discounted capital

$$
Y_{t}\left(\left\{\alpha, \omega_{\alpha}\right\}\right)=M^{1}\left\{\frac{f\left(S_{T}\left(\left\{\alpha, \omega_{\alpha}\right\}\right)\right)}{B_{0} \mathrm{e}^{r T}} \mid \mathcal{F}_{t}^{1}\right\}
$$

the representation

$$
\begin{gathered}
Y_{t}\left(\left\{\alpha, \omega_{\alpha}\right\}\right)= \\
=M_{\alpha}^{1} \frac{f\left(S_{T}\left(\left\{\alpha, \omega_{\alpha}\right\}\right)\right)}{B_{0} \mathrm{e}^{r T}}+\int_{[a, t]} \psi_{k(\alpha)}\left(\tau \mid \omega_{\alpha}\right) \mathrm{d} \bar{S}_{\tau}^{0}\left(\left\{\alpha, \omega_{\alpha}\right\}\right), t \in[a, b)
\end{gathered}
$$

is valid. Since

$$
X_{t}\left(\left\{\alpha, \omega_{\alpha}\right\}\right)=B_{0} \mathrm{e}^{r t} Y_{t}\left(\left\{\alpha, \omega_{\alpha}\right\}\right),
$$

then

$$
\begin{gathered}
X_{t}\left(\left\{\alpha, \omega_{\alpha}\right\}\right)=\mathrm{e}^{r t} \mathrm{e}^{-r T} M_{\alpha}^{1} f\left(S_{T}\left(\left\{\alpha, \omega_{\alpha}\right\}\right)\right) \\
+B_{0} \mathrm{e}^{r t} \int_{[a, t]} \psi_{k(\alpha)}\left(\tau \mid \omega_{\alpha}\right) \mathrm{d} S_{\tau}^{0}\left(\left\{\alpha, \omega_{\alpha}\right\}\right), t \in[a, b) .
\end{gathered}
$$

Taking into account the lemma 10, the definition of self-financing strategy, we obtain the proof of the theorem 6 .

\section{References}

1. Neveu J. Bases Mathematiques du Calcul des Probabilites. Paris, Masson et Cie, 1964.

2. Gonchar N.S. Financial Mathematics, Economic Growth. Kyiv, Rada, 2000 (in Russian). 


\section{Математична модель фондового ринку}

\section{М.С. Гончар}

Інститут теоретичної фізики ім. М.М.Боголюбова НАН України, 252143 Київ, вул. Метрологічна, $14^{\text {б }}$

Отримано 30 травня 2000 p.

В роботі побудовано математичну модель ринку цінних паперів. Отримані результати є доброю основою для аналізу подій на фондовому ринку.

Ключові слова: випадковий процес, ефективний ринок цінних паперів, оцінювання опціонів

PACS: $02.50 .+s, 05.40 .+j$ 